W. Meier, I. Boxx, M. Stöhr, C.D. Carter

Laser-based investigations in gas turbine model combustors

Experiments in Fluids 49:865-882 (2010)

18 Pages

The original publication is available at www.springerlink.com

http://dx.doi.org/10.1007/s00348-010-0889-x 


\title{
Laser-based investigations in gas turbine model combustors
}

\author{
W. Meier ${ }^{1}$, I. Boxx $^{1}$, M. Stöhr ${ }^{1}$, C.D. Carter $^{2}$ \\ ${ }^{1}$ Institut für Verbrennungstechnik, Deutsches Zentrum für Luft- und Raumfahrt (DLR), \\ Pfaffenwaldring 38, 70569 Stuttgart, Germany \\ ${ }^{2}$ Air Force Research Laboratory, Wright-Patterson Air Force Base, Dayton, OH 45433, USA \\ wolfgang.meier@dlr.de,Tel.: +497116862397,Fax:+497116862578
}

\begin{abstract}
Dynamic processes in gas turbine (GT) combustors play a key role in flame stabilization and extinction, combustion instabilities and pollutant formation, and present a challenge for experimental as well as numerical investigations. These phenomena were investigated in two gas turbine model combustors for premixed and partially premixed $\mathrm{CH}_{4} /$ air swirl flames at atmospheric pressure. Optical access through large quartz windows enabled the application of laser Raman scattering, planar laser-induced fluorescence (PLIF) of $\mathrm{OH}$, particle image velocimetry (PIV) at repetition rates up to $10 \mathrm{kHz}$ and the simultaneous application of $\mathrm{OH}$ PLIF and PIV at a repetition rate of $5 \mathrm{kHz}$. Effects of unmixedness and reaction progress in lean premixed GT flames were revealed and quantified by Raman scattering. In a thermoacoustically unstable flame, the cyclic variation in mixture fraction and its role for the feedback mechanism of the instability are addressed. In a partially premixed, oscillating swirl flame, the cyclic variations of the heat release and the flow field were characterized by chemiluminescence imaging and PIV, respectively. Using phase-correlated Raman scattering measurements, significant phase-dependent variations of the mixture fraction and fuel distributions were revealed. The flame structures and the shape of the reaction zones were visualized by planar imaging of $\mathrm{OH}$ distribution. The simultaneous OH PLIF/PIV high-speed measurements revealed the time history of the flow field-flame interaction and demonstrated the development of a local flame extinction event. Further, the influence of a precessing vortex core on the flame topology and its dynamics is discussed.
\end{abstract}

\section{Introduction}

Modern gas turbine (GT) combustors for power generation are frequently operated with lean premixed flames in order to avoid temperature peaks and high thermal $\mathrm{NO}_{\mathrm{x}}$ emissions. In most cases the flames are aero-dynamically stabilized by swirl, which leads to compact 
flames over a wide tuning range (Gupta et al. 1984). Swirl-induced vortex breakdown induces a recirculation of burned gas to the flame root and the mixing of hot gas and radicals with fresh gas leads to a continuous ignition and flame stabilization (Weber and Duguè 1992, Lucca-Negro and Doherty 2001). However, this process is prone to instabilities in the form of thermo-acoustic pulsations, unsteady stabilization, or even flame extinction. The dynamic processes of the flow field-flame interaction governing such behavior are complex and not well enough understood today. Periodic thermo-acoustic instabilities in lean premixed GT combustors pose a severe technical problem and often limit the operational range of GT engines and prevent further reduction of $\mathrm{NO}_{\mathrm{x}}$ emissions. It is well known that the pressure fluctuations are directly correlated with heat release variations which, in turn, are influenced by the flame stabilization mechanism. Several mechanisms responsible for instabilities have been identified (Lieuwen and Yang 2006), but the sequence of events sustaining the pulsations and the influence of the boundary conditions were in most cases not clearly identified.

One important aspect of flame stabilization and instabilities in lean premixed GT combustors is the effect of unmixedness and periodic variations of the equivalence ratio. In practical GT combustors, fuel is injected into the air flow shortly before entering the combustion chamber, and thus the time remaining for mixing is often not sufficient to allow for complete premixing (Meier et al. 2007, Ax et al. 2009, Stopper et al. 2010). The remaining unmixedness often improves the flame stabilization through near-stoichiometric regions but also limits the potential $\mathrm{NO}_{\mathrm{x}}$ reduction (Syed and Buchanan 2005). Dynamic pressure variations in the combustion chamber can impact the fuel and air flow rates and lead to periodic equivalence ratio oscillations that enhance and sustain the thermo-acoustic pulsations (Lieuwen et al. 2001, Sattelmayer 2003, Lawn et al. 2004, Muruganandam et al. 2005). These examples demonstrate the significance of the mixing process in GT combustors and underline the importance of appropriate measurement techniques for its temporally and spatially resolved determination.

Another important aspect is the reaction progress, which is closely related to flame stabilization. Finite-rate chemistry effects like local flame extinction or ignition delay are present in GT flames (Meier et al. 2006, Wehr et al. 2007) and can play a dominant role during the combustion process (Duan et al. 2004). However, it is difficult to measure these effects in turbulent flames. With respect to flow field-flame interactions, the flame dynamics are governed not only by the turbulent velocity field, but also by transient or periodic flow instabilities. Thermo-acoustic pulsations can lead to periodically changing flow structures 
such as vortex shedding (Schadow and Gutmark 1992, Kühlsheimer and Büchner 2002, Candel 2002, Lieuwen and Yang 2006) or oscillations of the inner recirculation zone, IRZ (Duan et al. 2005). Large-scale coherent structures such as the helical precessing vortex core (PVC) are often observed in swirling flows and flames (Fernandes et al. 2006, Schildmacher et al. 2006, Valera-Medina et al. 2009, Stöhr et al. 2009). Such structures contribute to the mixing process and interact with the flame front, leading to flame corrugation, stretch and local extinction. Since such interactions are highly dynamic and history-dependant, a thorough understanding of them requires simultaneous, time-resolved imaging of the flame sheet and the local turbulent flow-field.

A better understanding of the above mentioned aspects of GT combustion has been achieved in recent years by the application of laser measuring techniques (Eckbreth 1996, Wolfrum 1998, Kohse 2002, Kohse et al. 2005). The quantification of unmixedness and finite-rate chemistry effects in flames is possible by multi-species detection with laser Raman scattering (Grünefeld et al. 2000, Kojima and Nguyen 2004, Barlow 2007, Gregor et al. 2009, Ax et al. 2009). For example, the degree of unmixedness in a lean premixed industrial GT combustor was determined using this technique (Stopper et al. 2010) as was the phasedependent variation of the reaction progress in a partially premixed model GT combustor (Weigand et al. 2005). The interaction between the flow field and the flame front has been studied using various combinations of particle image velocimetry (PIV) and planar laser induced fluorescence (PLIF). The flame-vortex interaction was investigated by Meyer et al. applying OH PLIF and PIV in a counterflow flame (2004). Driscoll and coworkers applied PIV simultaneously with PLIF of $\mathrm{OH}$ or $\mathrm{CH}$ to characterize flame extinction phenomena in turbulent non-premixed jet flames (Carter et al. 1998, Donbar et al. 2001). Rehm and Clemens (1998) used PIV/OH PLIF and Han and Mungal (2003) used PIV/CH PLIF to study the influence of strain on the reaction zones in non-premixed jet flames. Watson et al. (1999) investigated the flame stabilization of a lifted $\mathrm{CH}_{4} /$ air diffusion flame by $\mathrm{CH}$ PLIF/PIV. Hult et al. (2005) used multi-shot, $\mathrm{kHz}$ frame-rate PLIF together with stereoscopic PIV to study the interaction between the reaction zone and turbulent vortices in the DLR jet flame. Similar approaches have been used in more complex GT-like flames as well. For example, Petersson et al. (2007) applied PIV, PLIF and filtered Rayleigh scattering to a premixed unconfined low swirl flame and Tanahashi et al. (2005) used CH and OH PLIF simultaneously with stereo PIV to study the flame structures in a confined swirl-stabilized premixed flame. Sadanandan et al. (2008) identified flow field-flame interactions in a GT-model combustor by OH PLIF and PIV. 
In most of these studies, the data rate was limited to around $10 \mathrm{~Hz}$; a notable exception is the work of the Lund group, wherein a multi-YAG cluster capable of generating up to 8 consecutive pulses in a short time was employed (Kaminski et al. 1999). In order to resolve the temporal development of the flow field or the flame structures in turbulent flames, repetition rates beyond $1 \mathrm{kHz}$ are needed, if possible over a sufficient duration to capture the full history of transient events like flame lift-off. With the development of high-speed CMOS cameras and diode pumped solid state lasers, in combination with frequency-doubled dye lasers, particle image velocimetry and planar laser-induced fluorescence measurements in flames became possible at frame-rates of $\geq 1 \mathrm{kHz}$ (Upatnieks et al. 2004, Wäsle et al. 2005, Kittler et al. 2007, Heeger et al. 2008, Fajardo and Sick 2009, Boxx et al. 2009a, Steinberg and Driscoll 2009). By the simultaneous application of PIV and PLIF of OH at frame-rates of $5 \mathrm{kHz}$ in a GT model combustor, Boxx et al. (2009b) demonstrated how the turbulent flow field and a precessing vortex core influenced the mixing process and the flame front structure. Alternative approaches based on different lasers sources have also been reported. Paa et al. (2007) used the frequency tripled radiation of a tunable disc laser around $343 \mathrm{~nm}$ for imaging $\mathrm{OH}$ in a $\mathrm{H}_{2} / \mathrm{O}_{2}$ diffusion flame at a frame-rate of $1 \mathrm{kHz}$. Very high repetition rates can be achieved over a short duration by the use of burst mode solid state lasers. In combination with an optical parametric oscillator, planar imaging of NO was performed in a supersonic flow (Jiang et al. 2009) and of $\mathrm{OH}$ in a turbulent diffusion flame (Miller et al. 2009) with frame-rates up to $50 \mathrm{kHz}$.

While the investigations have certainly resulted in a much better understanding of certain aspects of GT combustion and the underlying turbulence-chemistry interaction responsible for them, the resulting data is frequently unsuitable for direct comparison to numerical studies due, for example, to uncertainties in the boundary conditions or lack of sufficient experimental data. Alternately, they frequently do not adequately reproduce the complexity of GT combustors to meet the needs of engine developers. Thus, in recent years a need was recognized for a comprehensive database of high-resolution, multi-parameter experimental measurements of well-defined swirl flames under GT-relevant operating conditions with which to compare numerical and experimental results.

The DLR Institute of Combustion Technology has lead an ongoing effort to generate such a database through the use of a series of laboratory-scale gas turbine model combustors designed specifically for the purpose. The excellent optical access, compact size and moderate thermal load $(7-35 \mathrm{~kW})$ make these burners ideally suited to both point and planar laser measurement techniques. Each burner is operated in one of a series of well-defined, 
extensively characterized flow conditions and multiple, complimentary measurement techniques are applied. By using standard model combustors, it is possible to return to the same flame repeatedly for further investigation as new insight or improved measurement capabilities become available.

The intent of this paper is to illustrate the potential of contemporary laser measuring techniques for the investigation of GT-relevant combustion phenomena in two such model combustors; the Dual-Swirl Burner and the Turbomeca Burner. Examples will be given how laser Raman scattering, planar laser-induced fluorescence, chemiluminescence imaging, acoustic measurements and particle image velocimetry, applied separately or in combination with each other have enabled mutually reinforcing interpretations of the turbulence-chemistry interactions in these burners and contributed to a much deeper understanding of GT combustion phenomena in general.

\section{Measurement Techniques}

\subsection{Laser Raman scattering}

Quantitative point measurements of the major species concentrations $\left(\mathrm{O}_{2}, \mathrm{~N}_{2}, \mathrm{CH}_{4}, \mathrm{H}_{2}, \mathrm{CO}\right.$, $\mathrm{CO}_{2}, \mathrm{H}_{2} \mathrm{O}$ ) and the temperature were performed using laser Raman scattering. The radiation of a flashlamp-pumped dye laser (Candela LFDL 20, wavelength $\lambda=489 \mathrm{~nm}$, pulse energy $\mathrm{E}_{\mathrm{p}} \approx 3 \mathrm{~J}$, pulse duration $\tau_{\mathrm{p}} \approx 3 \mu \mathrm{s}$ ) was focused into the combustion chambers of the burners studied in this work; the Raman scattering emitted from the measuring volume (length $\approx 0.6$ $\mathrm{mm}$, diameter $\approx 0.6 \mathrm{~mm})$ was collected by an achromatic lens $(\mathrm{D}=80 \mathrm{~mm}, \mathrm{f}=160 \mathrm{~mm})$ and relayed to the entrance slit of a spectrograph (SPEX 1802, $\mathrm{f}=1 \mathrm{~m}$, slit width $2 \mathrm{~mm}$, dispersion $\approx 0.5 \mathrm{~nm} / \mathrm{mm}$ ). The dispersed and spatially separated signals from the different species were detected by individual photomultiplier tubes (PMTs) in the focal plane of the spectrograph and sampled using boxcar integrators. The species number densities were calculated from these signals using calibration measurements and the temperature was deduced from the total number density via the ideal gas law (Keck et al. 2002). The simultaneous detection of all major species with each laser pulse enabled the determination of the instantaneous mixture fraction $\xi$, defined as the mass fraction in the measurement volume that originates from the fuel nozzle, i.e. the contribution from $\mathrm{C}$ and $\mathrm{H}$ atoms to the total mass for $\mathrm{CH}_{4} /$ air combustion (Bergmann et al. 1998). At each measuring location, 400 single-shot measurements were acquired from which the joint probability density functions were computed. In the flames 
with self-excited thermo-acoustic pulsations, Raman measurements were also performed with phase-locking, i.e. the measuring system was triggered according to the phase-angle of the acoustic signal.

With respect to measurement uncertainties, one must distinguish between systematic errors arising from, for example, uncertainties in the calibration procedure, and statistical errors which are mainly caused by the statistics (shot noise) of the detected Raman photons in a single-shot measurement. Systematic uncertainties were typically $\pm 3-4 \%$ for the temperature $\mathrm{T}$ and mixture fraction, $\pm 3-5 \%$ for the mole fractions of $\mathrm{O}_{2}, \mathrm{H}_{2} \mathrm{O}$, and $\mathrm{CO}_{2}$, and $\pm 5-10 \%$ for $\mathrm{H}_{2}$ and $\mathrm{CO}$. In the measurements performed in the Turbomeca burner, it was determined that the evaluated $\mathrm{CH}_{4}$ mole fractions were systematically too large by about $7 \%$. The statistical uncertainties were quantified by recording data sets in stable laminar flames. The relative rms fluctuations in these flames were, for example, $2.5 \%$ for $\mathrm{T}$ at $1916 \mathrm{~K}, 3.2 \%$ for $\mathrm{H}_{2} \mathrm{O}$ at a mole fraction of $\mathrm{X}\left(\mathrm{H}_{2} \mathrm{O}\right)=0.19,7 \%$ for $\mathrm{O}_{2}$ at $\mathrm{X}\left(\mathrm{O}_{2}\right)=0.06,7.4 \%$ for $\mathrm{CO}_{2}$ at $\mathrm{X}\left(\mathrm{CO}_{2}\right)=0.068$, and $1 \%$ for the mixture fraction. A more detailed description of the measurement technique and uncertainties is given by Meier et al. (2007).

\subsection{OH PLIF system}

For the low-frame-rate OH PLIF measurements, a frequency doubled Nd:YAG laser (Quanta Ray, DCR-2) was used to pump a frequency doubled tunable dye laser (Lumonics, HD-500) at $10 \mathrm{~Hz}$. The UV beam $\left(\tau_{\mathrm{p}}=8 \mathrm{~ns}\right.$, line width $\left.\approx 0.4 \mathrm{~cm}^{-1}, \mathrm{E}_{\mathrm{p}}=2.5 \mathrm{~mJ}\right)$ was tuned to the $\mathrm{Q}_{1}(8)$ transition of $\mathrm{OH}$ at $\lambda \approx 283 \mathrm{~nm}$ in the $v^{\prime \prime}=0, v^{\prime}=1$ vibrational band of the $A^{2} \Sigma^{+}-X^{2} \Pi$ system. The population of the laser-coupled ground state of the selected line varies by approximately $9 \%$ in the temperature range of interest $(1400 \mathrm{~K}-2200 \mathrm{~K})$. OH fluorescence signal in the $v^{\prime}=1, v^{\prime \prime}=1$ and $v^{\prime}=0, v^{\prime \prime}=0$ bands near $310 \mathrm{~nm}$ was detected through a custom interference filter in the wavelength region 295-340 $\mathrm{nm}$. The laser sheet inside the combustor was approximately $50 \mathrm{~mm}$ in height and $400 \mu \mathrm{m}$ in thickness. By means of a beam splitter, a small portion of the incoming laser beam was directed into a $\mathrm{CH}_{4} /$ air reference flame stabilized on a McKenna burner. The resulting LIF was used for the online monitoring of the excitation line wavelength. The OH PLIF detection system consisted of an intensified CCD camera (Roper Scientific, 512 $\times 512$ pixels) with achromatic UV lens (Halle, f/2, f=64 mm). Part of incoming laser sheet was deflected into a quartz cell filled with fluorescent dye solution by means of a beam splitter. The dye cell fluorescence profile was simultaneously imaged using another ICCD camera (Roper Scientific, $512 \times 512$ pixels) equipped with a 50 
$\mathrm{mm}, \mathrm{f} / 1.8$ objective (Nikon). This dye cell profile was used for shot-to-shot correction of the laser sheet profile. The image intensifier was set to an exposure time of $400 \mathrm{~ns}$ for both the OH PLIF and dye cell fluorescence cameras. The spatial resolution of the system, based on the size of a pixel in the flow field, is approximately $185 \mu \mathrm{m}$. Further details of the experimental setup can be found in the literature (Sadanandan et al. 2008).

For the measurement of the chemiluminescence from electronically excited $\mathrm{OH}$ (denoted by $\mathrm{OH}^{*}$ ), an ICCD (LaVision, Imager Intense) equipped with an UV achromatic lens (Halle, $\mathrm{f} / 2, \mathrm{f}=100 \mathrm{~mm}$ ) and the same interference filter as for the LIF measurements was used. The exposure time was $40 \mu$ s for each image. In contrast to PLIF, chemiluminescence imaging is a line-of-sight integrating technique.

\subsection{PIV system}

The PIV system for low-frame-rate measurements (LaVision) consisted of a dual-cavity Nd:YAG laser $\left(\lambda=532 \mathrm{~nm}, \mathrm{E}_{\mathrm{p}}=120 \mathrm{~mJ}\right)$ and interline-transfer CCD camera with $1376 \mathrm{x} 1024$ pixels. The air flow of the flames was seeded with $\mathrm{TiO}_{2}$ particles of a nominal diameter of 1 $\mu \mathrm{m}$. The laser sheet had a thickness of $1 \mathrm{~mm}$ and was irradiated vertically through the central plane of the combustor. The laser sheet illuminated the entire chamber height $(110 \mathrm{~mm})$. Velocity fields were determined using a commercial multi-pass cross-correlation algorithm (LaVision DaVis 7.2). The spatial resolution of the velocity measurement was $1.1 \mathrm{~mm}$, based on the final interrogation window size of 16 pixels. The measuring uncertainty of instantaneous velocities was estimated as $0.4 \mathrm{~m} / \mathrm{s}$ based on the 0.1 pixel accuracy of the cross-correlation peak determination. Simultaneous recordings of the Q-switch trigger signal and the oscillating pressure signal from a microphone in the plenum were used to determine the phase angle of the individual PIV measurements with respect to the thermo-acoustic pulsation.

\subsection{Combined stereo-PIV/OH PLIF high-speed measuring system}

\subsubsection{OH PLIF system}

The OH PLIF system consisted of a modified conventional, frequency doubled dye laser and an intensified CMOS camera. The dye laser (Sirah Cobra-Stretch HRR, using Rhodamine 6G in ethanol, $0.06 \mathrm{~cm}^{-1}$ line width at $566 \mathrm{~nm}$ ) was pumped with a frequency doubled, diodepumped solid state $\mathrm{Nd}: \mathrm{YLF}$ laser (Edgewave IS-811E). At $5 \mathrm{kHz}$ repetition rate, the pump laser delivered $3.8 \mathrm{~mJ} /$ pulse at $523 \mathrm{~nm}$ with $8.5 \mathrm{~ns}$ pulse duration resulting in a frequency- 
doubled dye laser output of $0.1 \mathrm{~mJ} /$ pulse at $283.2 \mathrm{~nm}$. The wavelength was tuned to the peak of the $\mathrm{Q}_{1}(7)$ line of the $\mathrm{A}-\mathrm{X}\left(v^{\prime}=1, v^{\prime \prime}=0\right)$ transition of $\mathrm{OH}$, and the position was checked daily using a setup consisting of a laminar reference flame and a PMT for fluorescence detection. The $283.2 \mathrm{~nm}$ beam was formed into a sheet of $\approx 40 \mathrm{~mm}$ height in the combustion chamber using two fused silica lenses $\left(f_{p l i f 1}=-25 \mathrm{~mm}, f_{\text {plif2 }}=250 \mathrm{~mm}\right)$ in a cylindrical telescope configuration and focused to a waist of $\approx 400 \mu \mathrm{m}$ using a third cylindrical lens $\left(\mathrm{f}_{\text {plif3 }}=500 \mathrm{~mm}\right)$. The laser sheet was overlapped with the PIV laser sheet using a pair of dichroic mirrors (see Fig.1). The PIV and PLIF sheets were overlapped in the near and far fields and also in the probe region.

Fluorescence was acquired with a CMOS camera (LaVision HSS6), external two-stage, lens-coupled intensifier (LaVision HS-IRO) and UV objective (Cerco $\mathrm{f}=45 \mathrm{~mm}, \mathrm{f} / 1.8$ ). Background luminosity was reduced using a $500 \mathrm{~ns}$ intensifier gate and elastic (particle) scattering at $283 \mathrm{~nm}$ was blocked using a high-transmission ( $>80 \%$ at $310 \mathrm{~nm}$ ) bandpass interference filter (Laser Components $\mathrm{GmbH}$ ) and a color glass filter (1 mm thick WG295). Correction and normalization for spatial variation of the laser sheet intensity and camera/intensifier sensitivity were accomplished using a mean image derived from laserinduced fluorescence of acetone vapor in the combustion chamber. Run-to-run background and camera noise were corrected using a 1000 frame ensemble average acquired while the lasers were blocked.

\subsubsection{Particle Image Velocimetry}

The stereoscopic PIV system is based on a dual-cavity, diode-pumped, solid state Nd:YAG laser (Edgewave, IS-611DE, $\mathrm{E}_{\mathrm{p}}=2.6 \mathrm{~mJ}$ at $532 \mathrm{~nm}, \tau_{\mathrm{p}} \approx 14 \mathrm{~ns}$ ) and a pair of CMOS cameras (LaVision HSS5). Pulse timing separation for the PIV system was $\Delta \mathrm{t}=20 \mu \mathrm{s}$, with the $\mathrm{OH}$ PLIF excitation pulse temporally interlaced between the first and second PIV pulse.

As with the PLIF system, three cylindrical lenses were used to form the sheet: $f_{\text {pivl }}=-25$ $\mathrm{mm}, \mathrm{f}_{\mathrm{piv} 2}=300 \mathrm{~mm}$, and $\mathrm{f}_{\text {piv } 3}=1000 \mathrm{~mm}$. To minimize noise arising from inter-frame particle dropout, the beam waist was located beyond the probe region. The sheet thicknesses were measured to be $\leq 0.7 \mathrm{~mm}$ at the probe volume. Mie scattering from $\mathrm{TiO}_{2}$ particles seeded into the flow was imaged using the pair of CMOS cameras mounted equidistant from opposite sides of the laser sheet (see Fig.1). Each PIV camera had sufficient on-board memory (2.6 GB) for 4096 dual-frame images (i.e., 0.8 seconds imaging) at $512 \times 512$ pixel resolution. For each PIV camera, scattered light was collected with a $100 \mathrm{~mm}, \mathrm{f} / 5.6$ objective (Tokina), and 
image-blur due to off-axis defocusing was corrected using a Scheimpflug adaptor between the lens and camera. Perspective distortion was corrected using a dual-plane, three dimensional imaging target (LaVision Type 7). The same target was used to map the fields of view of the stereoscopic PIV and PLIF systems to one another. Image mapping, calibration, and particle cross-correlations were completed using a commercial, multi-pass adaptive window offset cross-correlation algorithm (LaVision $\mathrm{DaVis}$ 7.2). Final window size and overlap were $16 \times 16$ pixels and $50 \%$, respectively. This corresponds to a spatial resolution of $1 \mathrm{~mm}$ and vector spacing of $0.5 \mathrm{~mm}$. Based on the \pm 0.1 pixel accuracy of the crosscorrelation peak-finding algorithm, the absolute accuracy of the PIV measurements is estimated to be $\pm 0.3 \mathrm{~m} / \mathrm{s}$.

\subsection{Burners and combustion chambers}

\subsubsection{Turbomeca Burner}

This gas turbine model combustor was derived from an industrial design by Turbomeca. Fig.2 shows a schematic of the nozzle and combustion chamber. Dry air at ambient temperature is fed via a plenum $(\varnothing=78 \mathrm{~mm})$ through 12 radial swirler vanes to the burner nozzle. The fuel gas $\left(\mathrm{CH}_{4}\right)$ is injected into the air flow through small holes $(\varnothing=1 \mathrm{~mm})$ within the radial swirler with high momentum to allow good mixing before entering the combustion chamber. The nozzle exit has a diameter of $27.85 \mathrm{~mm}$. The air and fuel flows were each metered with electromechanical flow controllers (Brooks type 5853S for air and 5851S for $\mathrm{CH}_{4}$ ) and monitored using Coriolis flow meters (Danfoss Type 2100). The uncertainty of the flow measurements was $\pm 1.5 \%$ of the set flowrate. Above the nozzle is a square $(85 \times$ $85 \mathrm{~mm}$ ) combustion chamber, consisting of four $1.5 \mathrm{~mm}$ thick, $110 \mathrm{~mm}$ tall quartz windows, held at the corners by steel posts. The exit of the combustion chamber is a conical contraction leading to a short central exhaust pipe with a diameter of $40 \mathrm{~mm}$. The large windows on each side enable unobstructed optical access to nearly the entire flame zone. In order to change the measuring location within the flames, the burner could be translated in the axial and radial direction.

Two different flames were investigated in this burner. The first was an unsteady, pulsating (at about $290 \mathrm{~Hz}$ ) flame, operated at thermal power of $\mathrm{P}_{\mathrm{th}}=25 \mathrm{~kW}$ and an equivalence ratio of $\Phi=0.70$. For these operating conditions the Reynolds number at the exit of the nozzle, based on the cold flow and the exit diameter, is about 35000 . The second flame with $\mathrm{P}_{\mathrm{th}}=30 \mathrm{~kW}$ and $\Phi=0.83$ burned without strong thermo-acoustic pulsation. The shapes of the flame zone and flow field are indicated in Fig.2. The flow field is characterized by a 
conical shaped inflow of fresh gases, inner and outer recirculation zones and the corresponding shear layers.

\subsubsection{Dual swirl burner}

The second GT model combustor investigated in this study is shown schematically in Fig.3. Co-swirling dry air at room temperature was supplied to the flame through a central nozzle (diameter $15 \mathrm{~mm}$ ) and an annular nozzle (inner diameter $17 \mathrm{~mm}$, outer diameter $25 \mathrm{~mm}$ contoured to an outer diameter of $40 \mathrm{~mm}$ ). Both air flows were fed from a common plenum with an inner diameter of $79 \mathrm{~mm}$ and a height of $65 \mathrm{~mm}$. The radial swirlers consisted of 8 channels for the central nozzle and 12 channels for the annular nozzle. Non-swirling $\mathrm{CH}_{4}$ was fed through 72 channels $(0.5 \mathrm{~mm} \times 0.5 \mathrm{~mm})$ forming a ring between the air nozzles. The exit planes of the fuel and central air nozzles were located $4.5 \mathrm{~mm}$ below the exit plane of the outer air nozzle; the latter was defined as reference height $h=0$. The combustion chamber is the same as for the Turbomeca burner. As with the Turbomeca burner, the combustor was equipped with microphone probes (Brüel \& Kjaer, Type 4939) in the plenum and in a corner post of the combustion chamber. The microphone signals enabled the assignment of the laser and optical measurements to the phase angle of the acoustic pressure oscillations.

The flame described in this paper was operated at an air flow rate of 218 standard liters/min, slpm (referenced to STP conditions) and a fuel flow rate of 17.2 slpm corresponding to a thermal power of $\mathrm{P}_{\mathrm{th}}=10 \mathrm{~kW}, \Phi_{\text {global }}=0.75$ and a $\mathrm{Re}$ number of 15000 . Under these conditions it exhibited self-excited thermo-acoustic oscillations at a frequency of approximately $300 \mathrm{~Hz}$. The frequency could shift by about $\pm 3 \%$ between different measuring days due to changes in the ambient pressure by up to $\pm 2 \%$ and to uncertainties of the reproducibility of the flow rates. The mass flows of the gases were controlled by Brooks flow controllers (type 5853S for air and 5851S for $\mathrm{CH}_{4}$ ) and monitored via calibration-standard Coriolis mass flow meters (Siemens Sitrans-FC MassFlo 2100). Approximately 4\% of the air flow was delivered to the burner through a fluidized bed PIV particle seeder for the supply of $\mathrm{TiO}_{2}$.

The flame was not nozzle-attached, but rather oscillated chaotically about a lift-off height of several millimeters downstream. Thus, it was partially premixed before ignition. Fig.3 illustrates the shape of the flame zone and the main flow field characteristics, i.e., the inflow of fresh gases and the recirculation zones. Further details of the burner and flames can be found in previous publications (Weigand et al. 2006, Boxx et al. 2010). 


\section{Results and Discussion}

3.1 Mixing and reaction progress in the Turbomeca Burner

The results presented in this section are from laser Raman measurements in the Turbomeca burner. Fig.4 displays the correlation between the temperature and the mixture fraction for the non-pulsating $\left(\mathrm{P}_{\mathrm{th}}=30 \mathrm{~kW}\right)$ flame. Each symbol represents the result from a single laser shot recorded $6 \mathrm{~mm}$ above the burner nozzle and at various radial locations, denoted by the color of each symbol. For comparison, the solid line shows the calculated correlation for adiabatic equilibrium. The results reveal a significant scatter in mixture fraction, in particular in the inflow region, $\mathrm{r} \approx 13-16 \mathrm{~mm}$, where samples with mixture fractions ranging from $\xi \approx 0.03$ (corresponding to $\Phi \approx 0.53$ ) to $\xi \approx 0.075$ (corresponding to $\Phi \approx 1.39$ ) can be found. The global mixture fraction $\xi_{\text {global }}$ as derived from the mass flow rates is 0.0463 . This variation of mixture fraction demonstrates that a perfect premixing is not achieved in the short distance from the swirler to the nozzle exit (which corresponds to a convection time of approximately $2 \mathrm{~ms}$ ). Similar effects of unmixedness were also measured by Raman scattering in larger GT combustors and elevated pressure by Ax et al. (2009) and Stopper et al. (2010). It is noted that this flame (in contrast to that of the dual swirl combustor) is anchored below the nozzle exit, in particular in the shear layer between the IRZ and inflow, $r \approx 8-12 \mathrm{~mm}$, so that the flame "experiences" the variation in mixture fraction. However, the main region of heat release is further downstream $(\mathrm{h} \approx 30-60 \mathrm{~mm})$ where the mixtures are much closer to $\xi_{\text {global }}$; nonetheless, in this region there is still noticeable scatter in mixture fraction. In the IRZ and ORZ, the variation in $\xi$ is small. For example, in the IRZ, $\mathrm{r}=0-6 \mathrm{~mm}, \xi_{\text {mean }}$ is 0.047 with an RMS fluctuation of $2.5 \%$. This low variation in mixture fraction is explained by the relatively long residence time before the fluid elements reach the recirculation zones. Another striking result from Fig. 4 is the large variation in temperature. In the inlet region $(\mathrm{r} \approx 13-16 \mathrm{~mm})$, many samples exhibit room temperature, i.e., they have not reacted yet. In the IRZ, the samples are close to the equilibrium curve and can be regarded as completely reacted. A closer analysis of the compositions of the samples in the ORZ reveals that most of them are completely reacted but have experienced a temperature drop, probably due to heat loss at the burner plate or combustor wall. Many samples from the shear layer around $\mathrm{r}=10 \mathrm{~mm}$ have intermediate temperatures and a closer look at their compositions reveals that they contain exhaust gas as well as $\mathrm{CH}_{4}$ and $\mathrm{O}_{2}$, which means they are partially reacted. There are two reasons for this behavior: 1) local flame extinction has occurred, e.g., due to high strain rates, or 2) the 
mixtures are composed of hot exhaust gas from the recirculation zones and fresh gas that has not burned yet. From the analysis of OH PLIF measurements in this flame, it is clear that both effects, local flame extinction and mixing without reaction, are present in the flame (Meier et al. 2007).

To further illustrate the mixing and reaction progress, Fig.5 shows the corresponding correlation between the $\mathrm{CH}_{4}$ mole fraction and mixture fraction for the same samples. The solid line represents the state of pure mixing of $\mathrm{CH}_{4}$ and air without reaction and without admixtures of exhaust gas. Here again the large scatter in composition becomes obvious. The samples without $\mathrm{CH}_{4}$ are completely reacted and those with mole fractions between 0 and the state "mixing only" represent the partially reacted mixture with intermediate temperatures and the presence of $\mathrm{O}_{2}$.

At an equivalence ratio of 0.7 and a thermal power of $25 \mathrm{~kW}$ the flame exhibits thermoacoustic oscillations centered at a frequency of $\approx 290 \mathrm{~Hz}$. This oscillation has an additional influence on the mixing and reaction progress which was determined in phase-correlated measurements. Fig. 6 shows radial profiles of mixture fraction at the heights of 6 and $35 \mathrm{~mm}$ above the nozzle. Here, for each of the 8 phase angles, 400 single shot Raman measurements were averaged to reveal the phase-dependent variations. Phase 1 corresponded (by definition) to the minimum pressure in the plenum. At $\mathrm{h}=6 \mathrm{~mm}$, the phase-dependent changes are very small in the regions corresponding to the IRZ and ORZ. There is, however, a drastic change in the inflow region: At phase 2, which corresponds to the minimum pressure difference between the plenum and combustion chamber (Meier et al. 2007), the mixture fraction has the smallest value. This is mainly due to low $\mathrm{CH}_{4}$ concentrations exiting the burner nozzle at this phase angle (not to contributions from exhaust gas components). Thereafter, the mixture fraction increases gradually until phase 6 , followed by a steep rise at phase 7 and 8 where also the highest fuel concentrations were detected. At $\mathrm{h}=35 \mathrm{~mm}$, the phase-dependent variations of the mixture fraction have become smaller but are still clearly visible. At this height, the phase angles with the highest mean mixture fraction are 1 and 2 . This reflects a convective delay of approximately two phase-increments $\left(90^{\circ}\right)$ or $t \approx 0.86 \mathrm{~ms}$ as fluid propagates downstream from $\mathrm{h}=6 \mathrm{~mm}$ to $\mathrm{h}=35 \mathrm{~mm}$. The considerable change in mixture fraction has a significant influence on the combustion behavior, in particular on the heat release rate, as described by Meier et al. (2007). A detailed analysis of the data revealed the feedback mechanism of the periodic oscillation: During the period of large heat release, the pressure increases in the combustion chamber due to the thermal expansion and the pressure 
drop at the nozzle decreases. As a consequence the air flow rate is reduced significantly. However, the fuel flow rate at the injection holes in the swirler is much less reduced because the fuel is injected with a higher pressure drop than the air flow (Weigand et al. 2007). Thus, during the period of high pressure in the combustion chamber, high fuel concentrations accumulate in the swirler. With decreasing heat release rate in the combustion chamber and the associated drop in pressure, the fuel-rich $\mathrm{CH}_{4} /$ air mixtures enter the combustion chamber around phase 7 and 8 . When they reach the main flame zone at $\mathrm{h} \approx 40-60 \mathrm{~mm}$, the heat release increases again and the next cycle starts. The oscillation period of $3.45 \mathrm{~ms}$ is determined by the convection time from the swirler to the main flame zone. This feedback loop is known as an oscillating fuel supply combined with a convective time delay. The reason for the oscillating fuel supply lies in the different impedances of the fuel and air supply lines of the combustor. The detailed experimental results from these measurements yield a comprehensive and quantitative characterization of the flame behavior, thus making the data most suitable for the validation of numerical flame simulations (Galpin et al. 2008, Fiorina et al. 2010).

The findings above provide insight into the global dynamics of the combustor and elucidate the feedback mechanism responsible for sustaining thermo-acoustic pulsation. Their usefulness in identifying the underlying flow field-flame interactions responsible for the observed incomplete mixing and related phenomena is, however, limited. The mechanisms responsible for flame stabilization in this burner are highly dynamic and dependent on the instantaneous flow field and cannot be directly measured by the point measurements at $5 \mathrm{~Hz}$ repetition rate. In order to better understand the dynamic flow field of this burner, high-speed stereoscopic PIV measurements were recently performed on flames at the same run conditions listed above. The measurements were conducted in the plane of symmetry of the burner at a repetition rate of $10 \mathrm{kHz}$ over periods of $0.8 \mathrm{~s}$ per run. The field of view of was 38 $\mathrm{mm} \times 38 \mathrm{~mm}$ and was located directly above the nozzle. The acoustic frequency of the pulsating flame in this experiment was measured to be $\approx 280 \mathrm{~Hz}$, slightly lower than during the Raman measurements, and corresponding to a period of $\approx 3.57 \mathrm{~ms}$.

Figure 7 shows a sequence of 15 single-shot stereo PIV measurements. Here, only every third image of the $10 \mathrm{kHz}$ measurement is displayed in order to capture a full oscillation cycle without too many single images. The vectors represent the planar velocity components and the color bar indicates the velocity magnitude including the out-of-plane component. The prominent features of the flow field are the inflow region, the inner recirculation zone and 
several large-scale vortical structures in the shear layer between them. In the first image the reverse flow of the IRZ and incoming fresh gas from the nozzle form a local stagnation point at approximately $\mathrm{r} \approx 5 \mathrm{~mm}$ and $\mathrm{h} \approx 7 \mathrm{~mm}$. A similar stagnation-point flow configuration is seen one period later at $\mathrm{t}=3.6 \mathrm{~ms}$. At $\mathrm{t}=1.8 \mathrm{~ms}$, or half an acoustic cycle from frame 1 , the reverse flow is strong and extends below $\mathrm{h}=0$. A power spectrum of axial velocity taken at a monitor point in the IRZ indicates the change of the inflow velocity occurs periodically at the acoustic frequency of $280 \mathrm{~Hz}$.

In Fig.7, one also observes large-scale ( $\approx 10 \mathrm{~mm}$ diameter) vortical structures that appear to dominate parts of the flow field. The vortices appear regularly at the nozzle, e.g. at $\mathrm{t}=0.6$ $\mathrm{ms}$ at $\mathrm{r} \approx 6 \mathrm{~mm}$ and again at $\mathrm{t}=2.7 \mathrm{~ms}$, and propagate downstream along the inner shear layer. Although the field of view does not extend so far to the left side of the nozzle as to the right, vortices are also recognizable on this side, for example at $\mathrm{t}=2.4 \mathrm{~ms}$ and $\mathrm{t}=4.2 \mathrm{~ms}$. These have the opposite rotational direction and they are vertically displaced compared to the ones on the right side. This suggests that the vortices are part of a three-dimensional helical structure, probably a precessing vortex core. Numerical simulations for non-reacting flow in this burner predict the presence of a helical PVC with a precession frequency of $\approx 540 \mathrm{~Hz}$ (Roux et al. 2005). The same study however, also predicts this flow structure would be damped out in the presence of combustion. This appears not to be the case. Analysis of the high-speed PIV results confirmed that the asymmetric vortex-shedding observed in this sequence is very regular and periodic with a frequency of $\approx 570 \mathrm{~Hz}$. Thus, the instantaneous flow field may be seen as a superposition of two periodic phenomena, the PVC and the thermo-acoustic pulsation and overlaid by stochastic turbulent fluctuations. This makes the combustion behavior quite complex, and at the current state of data analysis, the interaction is not completely understood.

\subsection{Thermo-acoustic instabilities in the dual swirl burner}

The nozzle configuration and flame shape of this burner are quite different from the one of the Turbomeca burner, and the acoustic noise level of the instability was smaller than in the Turbomeca burner. The effective sound pressure of the oscillation measured in the combustion chamber was approximately $151 \mathrm{~Pa}$. Compared to the periodic pressure variation measured in the combustion chamber, the pressure measured in the plenum was delayed by $\approx 70^{\circ}$. Consistent with the definition of Duan et al., (2005), Phase $1\left(0^{\circ}\right)$ is defined as the pressure minimum in the plenum. Accordingly, the minimum pressure in the combustion 
chamber was at $\approx 290^{\circ}$ and the maximum at $\approx 110^{\circ}$. The measurements revealed that flame quantities like temperature, mixture fraction and heat release (determined from the $\mathrm{OH}^{*}$ chemiluminescence, as noted below) varied at the acoustic frequency.

Fig.8 displays the phase-correlated averages of the $\mathrm{OH}^{*}$ chemiluminescence and velocity distributions divided into bins of $45^{\circ}$ (phase angle). The chemiluminescence images are averaged over 60 to 65 single exposures for each phase angle. The chemiluminescence intensity reflects to a certain degree the heat release rate and its spatial distribution can be regarded as an indicator of the flame zone (Lee and Santavicca 2003, Hardalupas and Orain 2004). The images in Fig. 8 prove that the flame undergoes significant phase-dependent variations. The maximum overall chemiluminescence intensity appears around phase $4\left(135^{\circ}\right)$ and the minimum between phase 7 and 8 . Thus, the variation of the $\mathrm{OH}^{*}$ chemiluminescence intensity is closely in phase with the pressure variation in the combustion chamber, as expected from Rayleigh's criterion. The phase-correlated planar velocities shown in Fig.8 also exhibit significant phase-dependent variations with the highest inflow velocities appearing from phase 7 to 1 . Further, the shape of the distribution changes, for example the size of the recirculation zones, reflecting the flow field dynamics of the thermo-acoustic pulsation. A rough estimate of the convection time of a fluid element from the nozzle exit (taken as $\mathrm{h}=0, \mathrm{r} \approx 12 \mathrm{~mm}$ ) to the central region of the flame zone (taken as $\mathrm{h} \approx 17 \mathrm{~mm}$, $\mathrm{r} \approx 18 \mathrm{~mm}$ ), assuming a mean flow velocity of $\approx 10 \mathrm{~m} / \mathrm{s}$, yields $1.8 \mathrm{~ms}$, or approximately half of the thermo-acoustic oscillation period. The increased inflow of fuel/air mixtures from phase 7 to phase 1 might therefore be (partly) responsible for the increased heat release half a period later. However, it must also be considered that the thermal power and heat release rate are not directly correlated with the flow velocities at the nozzle, but rather with the mass flow rate of the fuel, which might behave differently from the bulk flow velocity. Finally, it is noted that the phase-correlated distributions of Fig. 8 indicate an axi-symmetric mean flow and flame structure in this burner.

Results from phase-correlated Raman measurements of mixture fraction and $\mathrm{CH}_{4}$ mole fraction are displayed in Fig.9 for the same phase angles as in Fig.8. In these 2D representations, the mean values from measurements at 45 locations in a grid pattern from $\mathrm{h}=5 \mathrm{~mm}$ to $\mathrm{h}=30 \mathrm{~mm}$ and $\mathrm{r}=0$ to $\mathrm{r}=30 \mathrm{~mm}$ are interpolated using an analysis / plotting program (Origin). Again, significant phase-dependent variations are apparent. The mixture fraction distributions reflect the degree of premixing and reveal that regions with a richer mixture are located near the fuel injector at $\mathrm{r}=8 \mathrm{~mm}$. Mixing proceeds rapidly with 
downstream position and the gas compositions are quite homogeneous for $h \geq 25 \mathrm{~mm}$. Around phase 5, when inflow velocities at the nozzle are close to the minimum, relatively fuel-rich mixtures appear at $\mathrm{h} \approx 5 \mathrm{~mm}$ that convect downstream, grow and finally dissipate. A comparison of the mixture fraction distributions with the $\mathrm{CH}_{4}$ distributions shows that their spatial development is quite similar from phase 5 to phase 2; however, at phases 3 and 4 discrepancies are apparent. This can be explained by an increased consumption of $\mathrm{CH}_{4}$, in agreement with the relatively high heat release rate at these phase angles seen in Fig.8. The measured temperatures and mole fractions are also in agreement with this finding. They show, for example, that the decrease in $\mathrm{CH}_{4}$ mole fraction coincides with an increase in temperature and $\mathrm{H}_{2} \mathrm{O}$ mole fraction.

The phase-correlated mean values reveal some important relationships between various thermodynamic quantities during an oscillation cycle: The variations of the heat release rate and the pressure in the combustion chamber are coupled and nearly in phase. As a consequence of the pressure variation, the inflow velocity changes periodically and, possibly due to different impedances of the fuel and air supplies, so does the mixture fraction. The increased volume flow at the nozzle is in agreement with the increase in heat release after a convective time delay of about half a period. However, to better understand the dynamics of this process, instantaneous flame structures and their temporal development must be considered.

Fig.10 shows a single-shot OH PLIF image recorded in the central vertical plane of the combustion chamber with the low-frame-rate system. The dark regions (i.e. those without $\mathrm{OH})$ represent the inflow of fresh gas and mixtures of fresh and burned gas at temperatures below $\mathrm{T} \approx 1500 \mathrm{~K}$. The highest $\mathrm{OH}$ LIF signals are attributed to super-equilibrium $\mathrm{OH}$ concentrations that are formed rapidly in the reaction zones. The decay towards equilibrium, however, takes a few milliseconds at atmospheric pressure (Sadanandan et al. 2008). OH is thus convected with the flow field for $10-30 \mathrm{~mm}$ at the flow velocities prevailing in the combustion chamber. At equilibrium, the $\mathrm{OH}$ concentration is typically a factor of 3-5 lower than the peak concentration levels. Of course, the local $\mathrm{OH}$ concentration is also governed by the temperature, and the LIF intensities are subject to electronic quenching effects. However, taking all influences into consideration, it becomes clear that the reaction zones are characterized by a steep increase in $\mathrm{OH}$ concentrations and that they can thus be identified by the large gradients of the $\mathrm{OH}$ PLIF distributions. The result of the gradient calculation is illustrated in the lower frame of Fig.10. Here, a Sobel gradient filter was used that takes the 
derivative in the one direction and smoothes in the orthogonal direction using a onedimensional version of triangular filter (Jähne 2005). The smaller gradients, for example those in the lower left corner of the image, probably represent a boundary layer between hot gas with $\mathrm{OH}$ close to equilibrium ("old $\mathrm{OH}^{\prime}$ ) and cold gas. From the shape of the large gradients and the $\mathrm{OH}$ distributions, it can be seen that the reaction zones are strongly contorted and that they are typically connected over a few centimeters. At some sections the flame segments are quite short indicating local flame extinction is an important factor. Furthermore, there are several islands of $\mathrm{OH}$. It is not clear whether these are part of a contiguous reaction zone connected to the main flame beyond the imaged plane or isolated pockets stemming from auto-ignition events. With respect to flame stabilization, simultaneous PIV and OH PLIF measurements revealed that the reaction zone is often located between the recirculating flow of hot products within the IRZ and the incoming flow of fresh gas (Sadanandan et al. 2008), resembling a stagnation flow flame.

There remain, however, several questions which cannot be answered by temporally noncorrelated single-shot images. This concerns, for example, the cause of local flame extinction (Böhm et al. 2009) or the evolution of coherent flow field structures and their influence on the flame front (Stöhr et al. 2009). Figure 11 shows a sequence of 15 consecutive images from simultaneous stereo PIV and OH PLIF measurements recorded at $5 \mathrm{kHz}$ repetition rate in the pulsating flame of the dual swirl burner. The field of view is $30 \mathrm{~mm}$ x $30 \mathrm{~mm}$ and lies above the nozzle, as indicated in the figure. As in Fig.10, the reaction zone is visualized by the large gradients extracted from the OH PLIF distributions. The vectors represent the inplane components of velocity, and the colors indicate the out-of-plane motion of the flow field. The main features of the flow field in the displayed field of view are the upstream motion within the IRZ and the inflow of fresh gas whose swirling motion can be identified by the opposite out-of-plane flow direction on the left and right side of the flame axis. As noted above the reaction zone is frequently located in or close to the boundary between the inflow and the reverse flow where the hot combustion products ignite the unburned gas mixtures. On the right hand side of the first image, a large vortex can be seen that moves upwards and to the right. The flame front is seen to wrap around this propagating vortex. At the same time, the flame front on the left hand side, which is roughly horizontally orientated becomes fainter from frame to frame and finally vanishes. The reason for this disappearance is likely local flame extinction resulting from high compressive strain rate at the flame front, which was determined to be on the order of $3000 \mathrm{~s}^{-1}$. At around image 6 another vortex appears at the left-hand side of the nozzle and subsequently propagates up and to the left. A few images 
later the flame surface engulfs the vortex, while simultaneously weakening noticeably on the right side of the field of view. A detailed evaluation revealed that the vortices are part of a three-dimensional helical vortex core that precesses through the shear layer dividing the inflow from the IRZ with a frequency of $\approx 510 \mathrm{~Hz}$. This fluid-dynamic frequency is not apparent in the measured acoustic signals. Rather, it was identified via a proper orthogonal decomposition of $\approx 4000$ planar velocity fields acquired in a given measurement run (Boxx et al. 2010).

Phase-averaging measurements with respect to only the thermo-acoustic oscillation results in axi-symmetric distributions like those seen in Figs.8 and 9. Subsequent analysis of the kHz-PIV / PLIF data, however, revealed that flame wrap-up by the PVC as observed in Fig.11 is a very repeatable, periodic phenomenon and results in a dynamically asymmetric flame. By sorting each measurement with respect to the phase of both the acoustic cycle and the PVC it was observed that while the flame is globally symmetric in the long-time mean, the instantaneous location and area of the flame surface (and thus heat release rate) is strongly affected by the PVC. The superposition of the $(\approx 510 \mathrm{~Hz})$ PVC precession frequency with the $(\approx 300 \mathrm{~Hz})$ thermo-acoustic oscillation results in a periodic axial expansion and contraction of the PVC and subsequent modification of the local turbulence field as it precesses around the centerline. The combined effect of these two phemonena is a periodic modulation of the flame front area and heat release rate at the thermo-acoustic frequency and a rotation of the centroid of the heat release around the burner axis at the difference frequency $\approx 210 \mathrm{~Hz}(510 \mathrm{~Hz}-300 \mathrm{~Hz})$. A more complete overview of the doubly phase-resolved analysis technique that revealed this turbulence-chemistry interaction will be reported in an upcoming paper (Steinberg et al. 2010).

Another interesting phenomenon observable in Fig. 11 is the occurrence of "OH islands". "OH islands" are small (1 - $2 \mathrm{~mm}$ diameter) regions of $\mathrm{OH}$ which suddenly appear in the imaging plane away from the identifiable, contiguous flame front. Such islands are observed frequently throughout each measurement run and tend to appear in the vicinity of the vortex cores, where they either grow rapidly and merge with the main flame front or quickly disappear. The origin of these isolated $\mathrm{OH}$ structures is not clear. They may represent narrow filaments of the main flame sheet, connected beyond the LIF imaging plane. Similar structures have been observed previously in premixed turbulent flames (Filatyev et al. 2005). Alternatively, they may be representative of isolated flame kernels resulting from autoignition near the burner exit. From the Raman measurements in this flame, it is known that 
mixtures of burned and unburned gases with relatively high temperatures are frequently present in this region; here, the ignition delay is expected to be on the order of $1 \mathrm{~ms}$ (Meier et al. 2006). The occurrence of the "OH islands" close to the vortex core, where strain rates are low and residence times are relatively long further supports this explanation. However, as LIF only indicates the presence of $\mathrm{OH}$ and not its source, the current measurements do not allow us to conclusively determine which mechanism leads to such "OH islands" in any specific instance.

\section{Summary and Conclusion}

Experimental investigations concerning major aspects of gas turbine (GT) combustion, including mixing and reaction progress, flame stabilization, finite-rate chemistry effects, and combustion instabilities, have been performed. The potential of laser diagnostic techniques in combination with optically accessible combustion chambers to improve understanding of GT phenomena was demonstrated. Observations from two GT model combustors, operated at atmospheric pressure with swirling $\mathrm{CH}_{4} /$ air flames up to $30 \mathrm{~kW}$ thermal power were presented. The laser techniques applied include spontaneous Raman scattering, planar laserinduced fluorescence (PLIF) of $\mathrm{OH}$ and particle image velocimetry (PIV) in combination with dynamic pressure measurements and chemiluminescence imaging. In particular, a system for long-duration, simultaneous OH PLIF/PIV with a sampling rate of $5 \mathrm{kHz}$ was presented.

The Raman measurements in the Turbomeca burner revealed a significant degree of unmixedness arising from the relatively short mixing time. The variation in mixture composition and the associated combustion temperature can be expected to have an impact on the $\mathrm{NO}_{\mathrm{x}}$ emissions and flame stabilization. Furthermore, the large variation in reaction progress demonstrated the significance of finite-rate chemistry effects. Apart from nonreacted and completely reacted samples, a large number of partially reacted mixtures were observed. These are explained by local flame extinction and mixing of burned and unburned gas without reaction. The measurements in the oscillating flame in the Turbomeca burner revealed a drastic phase-dependent variation of mixture fraction and corresponding cyclic variation of heat release. The results from phase-correlated Raman measurements formed the basis for understanding the feedback mechanism of the periodic instability and for the validation of numerical flame simulations. Stereoscopic PIV measurements at repetition rates of $10 \mathrm{kHz}$ revealed the dynamics of the flow field in this flame. Periodic variations of the 
recirculation zones and the inflow occurred at the acoustic frequency of $\approx 280 \mathrm{~Hz}$. In addition, coherent vortical structures, most likely representing a precessing vortex core (PVC) with a precession frequency of $\approx 570 \mathrm{~Hz}$, were identified in the shear layer. The interplay of the PVC with the thermo-acoustic oscillation and the flame dynamics is quite complex, of course, and thus still subject to ongoing analysis.

The phase-correlated measurements in the oscillating partially premixed flame in the dual-swirl burner showed that heat release, flow velocities, mixture fraction and fuel distributions varied at the acoustic frequency. Similar to the feedback mechanism of the oscillating Turbomeca flame, the results indicated that an oscillating fuel supply triggered by the pressure oscillation in the combustion chamber plays a major role for the sustainment of the pulsation. Simultaneous high-speed OH PLIF/PIV measurements revealed a complex underlying turbulence-chemistry interaction in the burner wherein a helical PVC with a frequency of $\approx 510 \mathrm{~Hz}$ plays a central role in flow field dynamics and results in a heat release distribution that is no longer axi-symmetric but rather moves around the flame axis. The long-duration planar kHz-PIV / PLIF measurements allow one to statistically condition the data on the phase of both the PVC and the acoustic signals, resulting in a more rigorous understanding of local flame dynamics.

The high-speed PIV and the simultaneous high-speed OH PLIF/PIV measurements provided new insights into the dynamics of turbulent swirl flames far beyond the possibilities of "slow" imaging techniques. The ability to resolve the temporal development of the flow field-flame interaction enabled the visualization of processes like local flame extinction and the interaction of a propagating vortex with the flame front. The results from the high-speed imaging measurements presented here were mostly discussed phenomenologically. However, the PIV measurements yield quantitative data and enable the determination of, e.g., vorticity or strain rates. Further, the long run-time allowed for the accumulation of several thousand consecutive images per run, corresponding to $\approx 250$ cycles of the thermoacoustic oscillation in the flames discussed here. This large number of images enables a detailed statistical data analysis, for example an averaging conditioned on the acoustic frequency and/or precessing vortex core frequency.

It must be kept in mind that swirl-stabilized flames are highly three-dimensional and dynamic. Although stereo PIV enables the determination of the out-of-plane velocity, the orientation and movement of the flame front with respect to the laser sheet cannot be evaluated from the measurements shown. Therefore it is not possible to deduce a flame 
displacement speed from the results (Tanahashi et al. 2008, Hartung et al. 2009) or to distinguish between flame hole mending by flame propagation and out-of-plane convection of the flame hole (Boxx et al. 2009a). An interesting open question concerns also the frequent appearance of isolated $\mathrm{OH}$ regions in the presented results. It seems plausible that these events stem from auto-ignition events; however, with the setup used in the presented experiments, it cannot be excluded that the $\mathrm{OH}$ pockets were convected from regions in front of or behind the laser sheet. High speed measurements with an experimental arrangement using an additional plane of OH PLIF are currently underway and may better answer this question.

\section{Acknowledgements}

The authors thank Rajesh Sadanandan for his contribution to OH LIF measurements, Adam Steinberg for the analysis of part of the high-speed measurements and Peter Weigand and Xuru Duan for the participation in the Raman experiments. The financial support within the DLR project MVS is gratefully acknowledged.

\section{References}

Ax H, Stopper U, Meier W, Aigner M, Güthe F (2009) Experimental analysis of the combustion behavior of a gas turbine burner by laser measurement techniques. In: Proceedings of the ASME Turbo Expo, Orlando, USA, GT2009-59171

Barlow RS (2007) Laser diagnostics and their interplay with computations to understand turbulent combustion. Proc Combust Inst 31:49-75

Bergmann V, Meier W, Wolff D, Stricker W (1998) Application of spontaneous Raman and Rayleigh scattering and 2D LIF for the characterization of a turbulent $\mathrm{CH}_{4} / \mathrm{H}_{2} / \mathrm{N}_{2}$ jet diffusion flame. Appl Phys B 66: 489-502

Böhm B, Heeger C, Boxx I, Meier W, Dreizler A (2009) Time-resolved conditional flow field statistics in extinguishing turbulent opposed jet flames using simultaneous highspeed PIV/OH PLIF. Proc Combust Inst 32:1647-1654

Boxx I, Heeger C, Gordon R, Böhm B, Dreizler A, Meier W (2009a) On the importance of temporal context in interpretation of flame discontinuities. Combust Flame 156:269-271

Boxx I, Stöhr M, Carter C, Meier W (2009b) Sustained multi-kHz flamefront and 3component velocity-field measurements for the study of turbulent flames. Appl Phys B 95:23-29 
Boxx I, Stöhr M, Carter C, Meier W (2010) Temporally resolved planar measurements of transient- and temporally developing phenomena in a partially pre-mixed swirl flame in a gas turbine model combustor. Combust Flame, accepted

Candel S (2002) Combustion dynamics and control: Progress and challenges. Proc Combust Inst 29:1-28

Carter CD, Donbar JM, Driscoll JF (1998) Simultaneous CH planar laser-induced fluorescence and particle imaging velocimetry in turbulent nonpremixed flames. Appl Phys B 66:129-132

Donbar JM, Driscoll JF, Carter CD (2001) Strain rates measured along the wrinkled flame contour within turbulent non-premixed jet flames. Combust Flame 125:1239-1257

Duan XR, Weigand P, Meier W, Keck O, Lehmann B, Stricker W, Aigner M (2004) Experimental investigations and laser based validation measurements in a gas turbine model combustor. Prog Comp Fluid Dynamics 4:175-182

Duan XR, Meier W, Weigand P, Lehmann B (2005) Phase-resolved laser Raman scattering and laser Doppler velocimetry applied to periodic instabilities in a gas turbine model combustor. Appl Phys B 80:389-396

Eckbreth AC (1996) Laser diagnostic for combustion temperature and species. Gordon and Breach, Australia

Fajardo C, Sick V (2009) Development of a high-speed UV particle image velocimetry technique and application for measurements in internal combustion engines. Exp Fluids $46: 43-53$

Fernandes EC, Heitor MV, Shtork SI (2006) An analysis of unsteady highly turbulent swirling flow in a model vortex combustor. Exp Fluids 40:177-187

Filatyev SA, Driscoll JF, Carter CD, Donbar JM (2005) Measured properties of turbulent premixed flames for model assessment, including burning velocities, stretch rates, and surface densities. Combust Flame 141:1-21

Fiorina B, Vicquelin R, Auzillon P, Darabiha N, Gicquel O, Veynante D (2010) A filtered tabulated chemistry model for LES of premixed combustion. Combust Flame 157:465-475

Galpin J, Naudin A, Vervisch L, Angelberger C, Colin O, Domingo P (2008) Large-eddy simulation of a fuel-lean premixed turbulent swirl-burner. Combust Flame 155:247-266

Gregor MA, Seffrin F, Fuest F, Geyer G, Dreizler A (2009) Multi-scalar measurements in a premixed swirl burner using 1D Raman/Rayleigh scattering. Proc Combust Inst 33:17321746

Grünefeld G, Schütte M, Andresen P (2000) Simultaneous multiple-line Raman/Rayleigh/LIF measurements in combustion. Appl Phys B 70:309-313 
Gupta AK, Lilley DG, Syred N (1984) Swirl flows. Abacus Press, Kent, UK

Han D, Mungal MG (2003) Simultaneous measurement of velocity and CH distributions. Part 1: jet flames in co-flow. Combust Flame 132:565-590

Hardalupas Y, Orain M (2004) Local measurements of the time-dependent heat release rate and equivalence ratio using chemiluminescent emission from a flame. Combust Flame 139: $188-207$

Hartung G, Hult J, Balachandran R, Mackley MR, Kaminski CF (2009) Flame front tracking in turbulent lean premixed flames using stereo PIV and time-sequenced planar LIF of OH. Appl Phys B 96:843-862

Heeger C, Böhm B, Boxx I, Meier W, Ahmed SF, Mastorakos E, Dreizler A (2008) Planar laser diagnostics at high repetition rates: Acquisition and analysis of transient combustion processes. Proceedings of the ASME Turbo Expo, Berlin, Germany, Paper GT2008-50152

Hult J, Meier U, Meier W, Harvey A, Kaminski CF (2005) Experimental analysis of local flame extinction in a turbulent jet diffusion flame by high repetition 2D laser techniques and multiscalar measurements. Proc Combust Inst 30:701-709

Jähne B (2005) Digital image processing. Springer, Berlin, Germany

Jiang N, Webster MC, Lempert WR (2009) Advances in generation of high-repetition-rate burst mode laser output. Appl Opt 48:B23-B31

Kaminski CF, Hult J, Aldèn M (1999) High repetition rate planar laser induced fluorescence of $\mathrm{OH}$ in a turbulent non-premixed flame. Appl Phys B 68:757-760

Keck O, Meier W, Stricker W, Aigner M (2002) Establishment of a confined swirling natural gas/air flame as a standard flame: Temperature and species distributions from laser Raman measurements. Combust Sci Technol 174:117-151

Kittler C, Dreizler A (2007) Cinematographic imaging of hydroxyl radicals in turbulent flames by planar laser-induced fluorescence up to $5 \mathrm{kHz}$ repetition rate. Appl Phys B 89:163-166

Kohse-Höinghaus K, Jeffries, J (eds) (2002) Applied combustion diagnostics. Taylor and Francis, New York, USA

Kohse-Höinghaus K, Barlow RS, Aldén M, Wolfrum J (2005) Combustion at the focus: laser diagnostics and control. Proc Combust Inst 30:89-123

Kojima J, Nguyen Q-V (2004) Measurement and simulation of spontaneous Raman scattering in high-pressure fuel-rich $\mathrm{H}_{2}$-air flames. Meas Sci Technol 15:565-580

Külsheimer C, Büchner H (2002) Combustion dynamics of turbulent swirling flames. Combust. Flame 131:70-84 
Lawn CJ, Evesque S, Polifke W (2004) A model for the thermoacoustic response of a premixed swirl burner, Part I: Acoustic aspects. Combust Sci Technol 176:1331-1358

Lee JG, Santavicca DA (2003) Experimental diagnostics for the study of combustion instabilities in lean premixed combustors. J Propulsion Power 19:735-750

Lieuwen T, Torres H, Johnson C, Zinn BT (2001) A mechanism of combustion instability in lean premixed gas turbine combustors. Transaction of the ASME 123:182-189

Lieuwen TC, Yang V (2006) Combustion instabilities in gas turbine engines. American Institute of Aeronautics and Astronautics, Inc. Reston, Virginia

Lucca-Negro O, Doherty TO (2001) Vortex breakdown: A review. Prog Energy Combust Sci 27:431-481

Meier W, Weigand P, Duan XR, Giezendanner-Thoben R (2007) Detailed characterization of the dynamics of thermoacoustic pulsations in a lean premixed swirl flame. Combust Flame 150:2-26

Meier W, Duan XR, Weigand P (2006) Investigations of swirl flames in a gas turbine model combustor II. Flow field, structures, temperature, and species distributions. Combust Flame 144: 225-236

Meyer TR, Fiechtner GJ, Gogineni SP, Rolon JC, Carter CD, Gord JR (2004) Simultaneous PLIF/PIV investigation of vortex-induced annular extinction in $\mathrm{H}_{2}$-air counterflow diffusion flames. Exp Fluids 36:259-267

Miller JD, Slipchenko M, Meyer TR, Jiang N, Lempert WR, Gord JR (2009) Ultrahigh-framerate $\mathrm{OH}$ fluorescence imaging in turbulent flames using a burst-mode optical parametric oscillator. Opt Lett 34:1309-1311

Muruganandam TM, Kim B-H, Moreell MR, Nori V, Patel M, Romig BW, Seitzman JM (2005) Optical equivalence ratio sensors for gas turbine combustors. Proc Combust Inst 30:16011609.

Paa W, Müller D, Stafast H, Triebel W (2007) Flame turbulences recorded at $1 \mathrm{kHz}$ using planar induced fluorescence upon hot band excitation of $\mathrm{OH}$ radicals. Appl Phys B 86:1-5

Petersson P, Olofsson J, Brackmann C, Seyfried H, Zetterberg J, Richter M, Alden M, Linne MA, Cheng RK, Nauert A, Geyer D, Dreizler A (2007) Simultaneous PIV/OH-PLIF, Rayleigh thermometry/OH-PLIF and stereo PIV measurements in a low-swirl flame. Appl Opt 46:3928-3936

Rehm JE, Clemens NT (1998) The relationship between vorticity/strain and reaction zone structure in turbulent nonpremixed jet flames. Proc Comb Inst 27:1113-1120 
Roux S, Lartigue G, Poinsot T, Meier U, Bérat C (2005) Studies of mean and unsteady flow in a swirled combustor using experiments, acoustic analysis, and large eddy simulations. Combust Flame 141:40-54

Sadanandan R, Stöhr M, Meier W (2008) Simultaneous OH PLIF and PIV measurements in a gas turbine model combustor. Appl Phys B 90:609-618

Sattelmayer T (2003) Influence of the combustor aerodynamics on combustion instabilities from equivalence ratio fluctuations. J. Eng. Gas Turbines Power 125:11-19

Schadow KC, Gutmark E (1992) Combustion instability related to vortex shedding in dump combustors and their passive control. Prog Energy Combust Sci 18:117-132

Schildmacher K-U, Koch R, Bauer HJ (2006) Experimental characterization of premixed flame instabilities of a model gas turbine burner. Flow Turbul Combust 76:177-197

Steinberg AM, Driscoll JF (2009) Straining and wrinkling processes during turbulencepremixed flame interaction measured using temporally-resolved diagnostics. Combust Flame 156: 2285-2306

Steinberg AM, Boxx I, Stöhr M, Carter CD, Meier W (2010) Flow-flame interactions causing acoustically coupled heat release fluctuations in a thermo-acoustically unstable gas turbine model combustor. Combust Flame, submitted

Stöhr M, Sadanandan R, Meier W (2009) Experimental study of unsteady flame structures of an oscillating swirl flame in a gas turbine model combustor. Proc Combust Inst 32:29252932

Stopper U, Aigner M, Ax H, Meier W, Sadanandan R, Stöhr M, Bonaldo A (2010) PIV, 2DLIF and 1D-Raman measurements of flow field, composition and temperature in premixed gas turbine flames. Exp Thermal Fluid Sci 34:396-403

Syed KJ, Buchanan E (2005) The nature of NOx formation within an industrial gas turbine dry low emission combustor. In. Proceedings of the ASME Turbo Expo, Reno-Tahoe, Nevada, USA, GT-2005-68070

Tanahashi M, Muratami S, Choi G-M, Fukuchi Y, Miyauchi T (2005) Simultaneous CH-OH PLIF and Stereoscopic PIV Measurements of Turbulent Premixed Flames. Proc Combust Inst 30:1665-1672

Tanahashi M, Taka S, Shimura M, Miyauchi T (2008) CH double-pulsed PLIF measurements in turbulent premixed flame. Exp Fluids 45:323-332 
Upatnieks A, Driscoll JF, Rasmussen CC, Ceccio SL (2004) Liftoff of turbulent jet flamesassessment of edge flame and other concepts using cinema-PIV. Combust Flame 138:259272

Valera-Medina A, Syred N, Griffiths A (2009) Visualisation of isothermal large coherent structures in a swirl burner. Combust Flame 159:1723-1734

Wäsle J, Winkler A, Sattelmayer T (2005) Spatial coherence of the heat release fluctuations in turbulent jet and swirl flames. Flow Turbul Combust 75:29-50

Watson KA, Lyons KM, Donbar JM, Carter CD (1999) Scalar and velocity field measurements in a lifted $\mathrm{CH}_{4}$-air diffusion flame. Combust Flame 117:257-271

Weber R, Dugué J (1992) Combustion accelerated swirling flows in high confinements. Prog Energy Combust Sci 18:349-367

Wehr L, Meier W, Kutne P, Hassa C (2007) Single-pulse 1D laser Raman scattering applied in a gas turbine model combustor at elevated pressure. Proc Comb Inst 31:3099-3106

Weigand P, Meier W, Duan XR, Giezendanner-Thoben R, Meier U (2005) Laser diagnostic study of the mechanism of a periodic combustion instability in a gas turbine model combustor. Flow Turbul Combust 75:275-292

Weigand P, Meier W, Duan XR, Stricker W, Aigner M (2006) Investigations of swirl flames in a gas turbine model combustor. Part I: flow field, structures, temperature and species distributions. Combust Flame 144:205-224

Weigand P, Meier W, Duan XR, Aigner M (2007) Laser-based investigations of thermoacoustic instabilities in a lean premixed gas turbine model combustor. J Eng Gas Turbine Power 129: 664-671

Wolfrum J (1998) Lasers in combustion: From basic theory to practical devices. Proc Combust Inst 28:1-41

\section{Figure captions}

Fig.1 Setup for the simultaneous high-speed $\mathrm{OH}$ PLIF/PIV measurements at $5 \mathrm{kHz}$ repetition rate.

Fig.2 Schematic drawing of the Turbomeca premixed swirl burner. The converging nozzle has a conical bluff-body on the axis. The shape of the flame zone is indicated in the combustion chamber and the overall flow field is sketched by the arrows.

Fig.3 Schematic drawing of the dual swirl burner. The sketch in the combustion chamber indicates the inner (IRZ) and outer (ORZ) recirculation zones and the average shape of the oscillation flame. 
Fig.4 Scatterplot of temperature vs mixture fraction from Turbomeca burner. Symbols represent results from single-shot measurements from various radial locations. The solid curve shows for comparison the state of adiabatic equilibrium. The vertical line shows the global mixture fraction corresponding to $\Phi=0.83$. The stoichiometric mixture fraction for $\mathrm{CH}_{4} /$ air flames is 0.055 .

Fig.5 Scatterplot of $\mathrm{CH}_{4}$ mole fraction vs mixture fraction from Turbomeca burner. The solid line represents the state of pure mixing of $\mathrm{CH}_{4}$ and air without reaction or admixtures of exhaust gas.

Fig.6 Radial profiles of phase-related mean mixture fraction for 8 equidistant phase angles. At each measuring location, 400 single shots were averaged for each phase angle of the acoustic oscillation.

Fig.7 Image sequence from stereo PIV measurements in acoustically unstable flame of the Turbomeca burner. Every $3^{\text {rd }}$ frame of the $10 \mathrm{kHz}$ measurement is displayed. The vectors represent the planar velocity ( $\mathrm{x}$ and $\mathrm{y}$ component), the color bar represents the velocity magnitude (including also the $\mathrm{z}$ component). The flame axis is at $\mathrm{x}=0$. The nozzle exit is indicated in the frames at $\mathrm{t}=3.6$ and $3.9 \mathrm{~ms}$.

Fig. 8 Phase-correlated mean distributions of the $\mathrm{OH}$ chemiluminescence and planar velocities from the dual swirl burner for 8 phase angles.

Fig.9 Phase-correlated mean distributions of mixture fraction (left half of each image) and $\mathrm{CH}_{4}$ mole fraction (right half of each image) from the dual-swirl burner. The results from Raman measurements in a grid of 45 locations have been interpolated to create 2D plots.

Fig.10 Single shot OH PLIF image (upper frame) recorded in the oscillating flame $\left(\mathrm{P}_{\mathrm{th}}=10\right.$ $\mathrm{kW}$ ) in the dual swirl burner and corresponding gradients of the OH PLIF distribution.

Fig.11 Image sequence from simultaneous OH PLIF/PIV measurements in the dual-swirl burnerat $5 \mathrm{kHz}$ frame-rate. The flame front can be identified by the large gradients of the $\mathrm{OH}$ LIF distributions. Vectors show in-plane velocity components, the colors of the vectors indicate the out-of-plane velocity component according to the color bar. The field of view is $30 \mathrm{~mm} \times 30 \mathrm{~mm}$. The position and shape of the nozzle are shown below the distributions. 


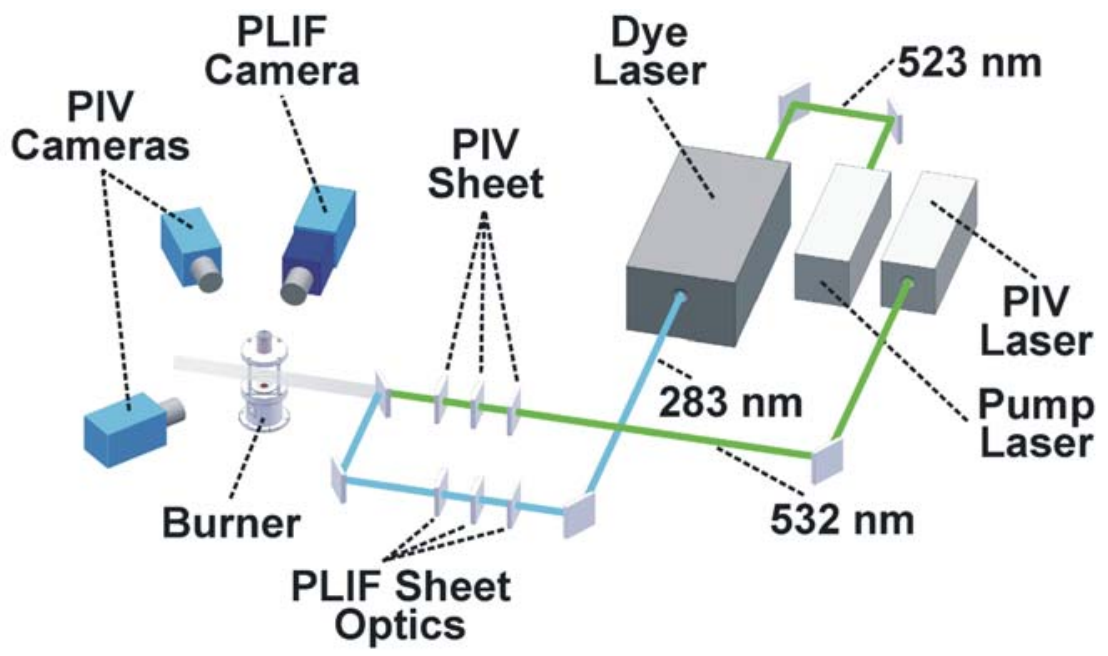

Fig.1 Setup for the simultaneous high-speed OH PLIF/PIV measurements at $5 \mathrm{kHz}$ repetition rate.

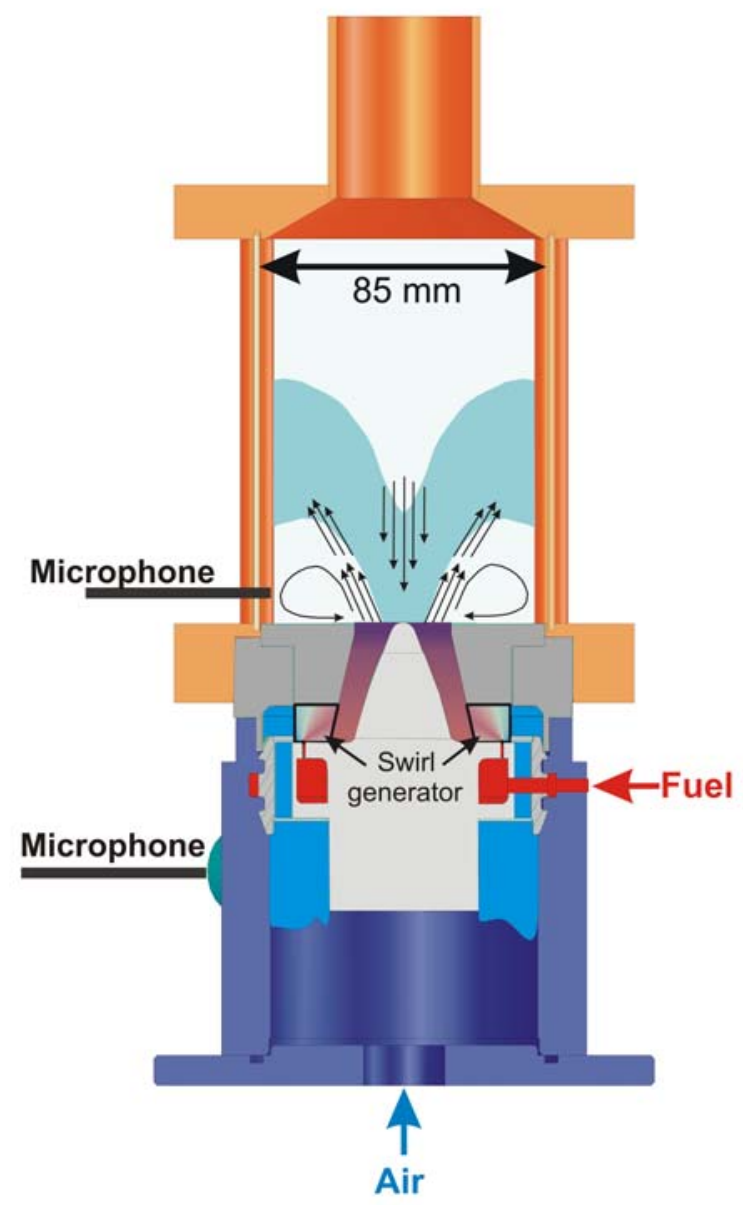

Fig.2 Schematic drawing of the Turbomeca premixed swirl burner. The converging nozzle has a conical bluff-body on the axis. The shape of the flame zone is indicated in the combustion chamber and the overall flow field is sketched by the arrows. 


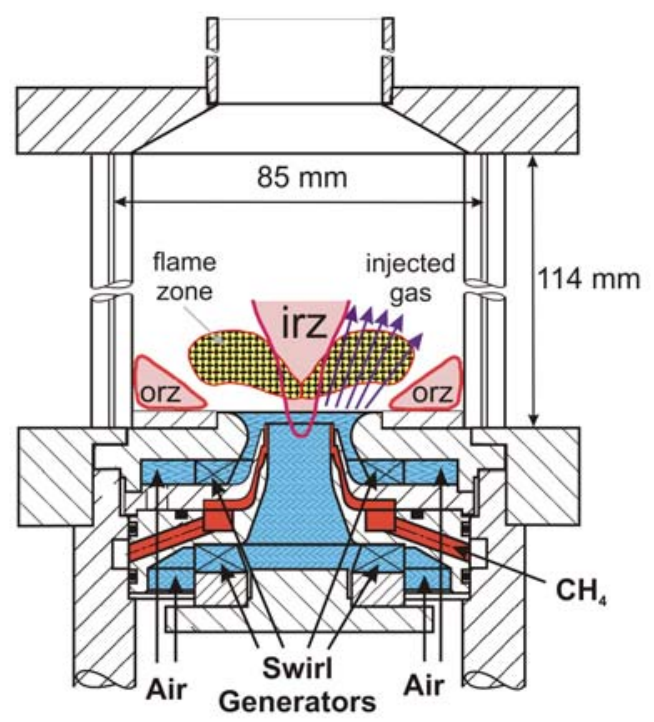

Fig.3 Schematic drawing of the double swirl burner. The sketch in the combustion chamber indicates the inner (IRZ) and outer (ORZ) recirculation zones and the average shape of the oscillation flame.

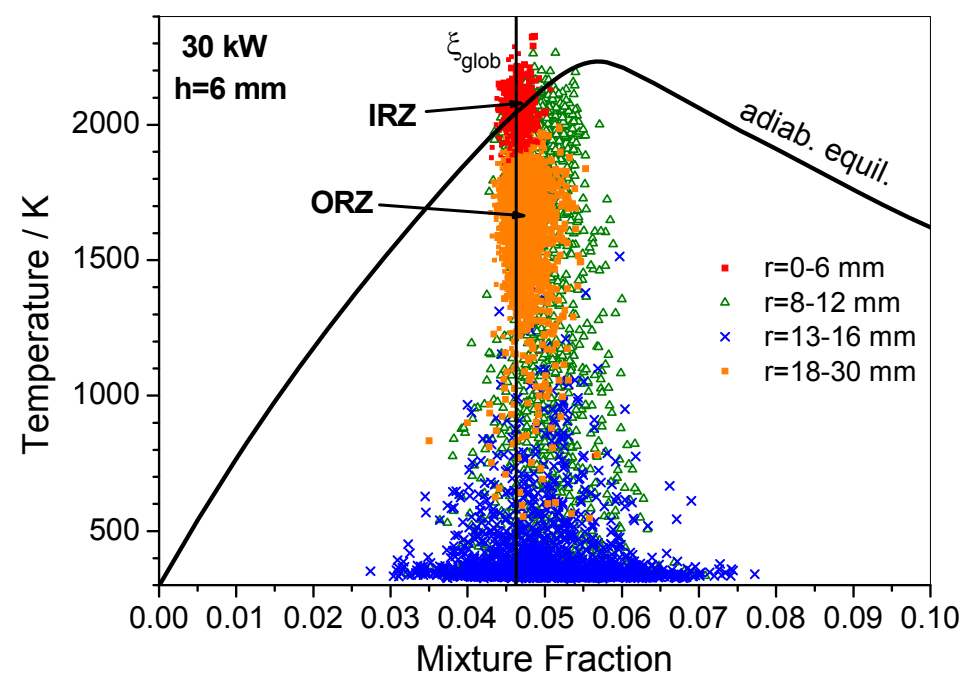

Fig.4 Scatterplot of temperature vs mixture fraction from Turbomeca burner. Symbols represent results from single-shot measurements from various radial locations. The solid curve shows for comparison the state of adiabatic equilibrium. The vertical line shows the global mixture fraction corresponding to $\Phi=0.83$. The stoichiometric mixture fraction for $\mathrm{CH}_{4}$ /air flames is 0.055 . 


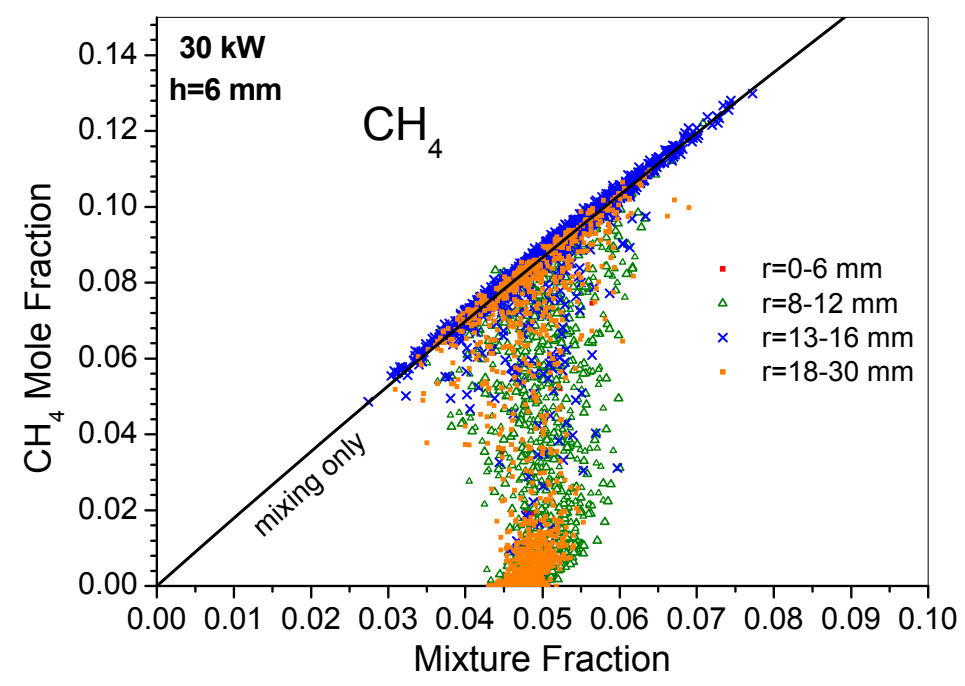

Fig.5 Scatterplot of $\mathrm{CH}_{4}$ mole fraction vs mixture fraction from Turbomeca burner. The solid line represents the state of pure mixing of $\mathrm{CH}_{4}$ and air without reaction or admixtures of exhaust gas.

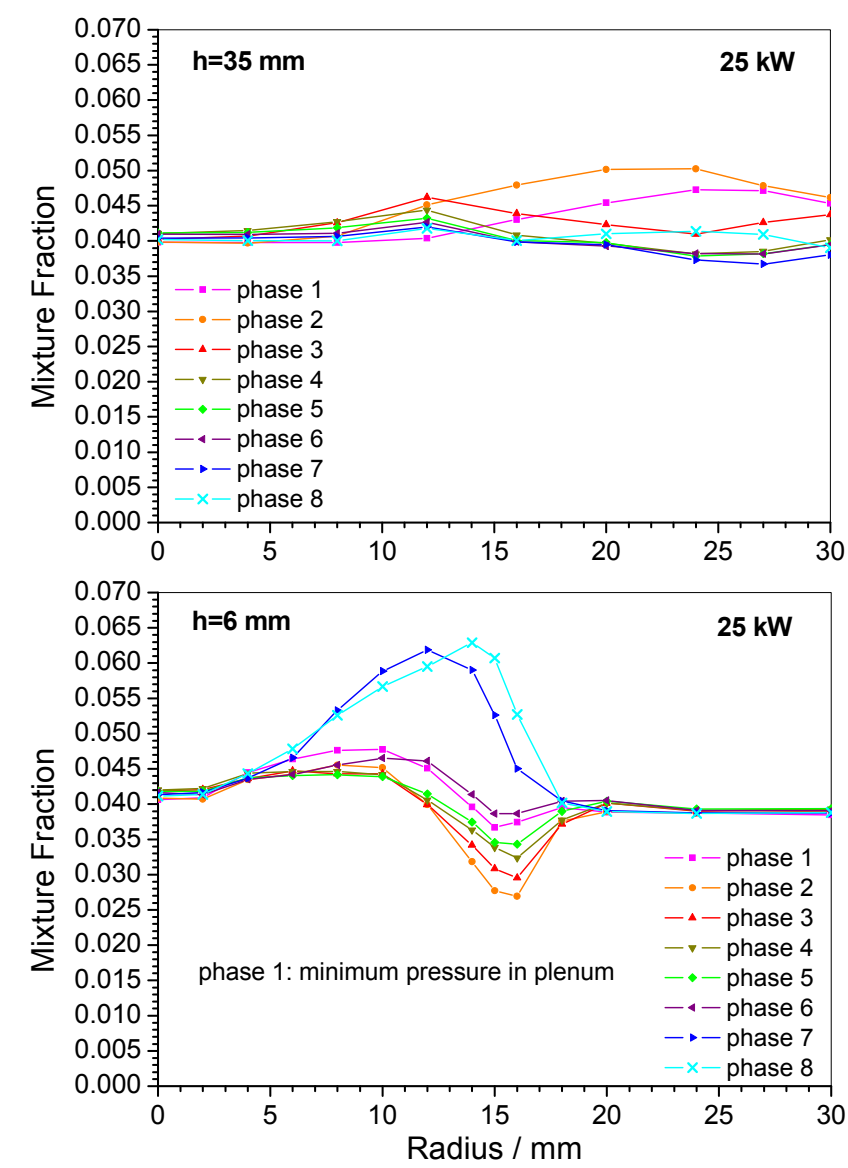

Fig.6 Radial profiles of phase-related mean mixture fraction for 8 equidistant phase angles. At each measuring location, 400 single shots were averaged for each phase angle of the acoustic oscillation. 

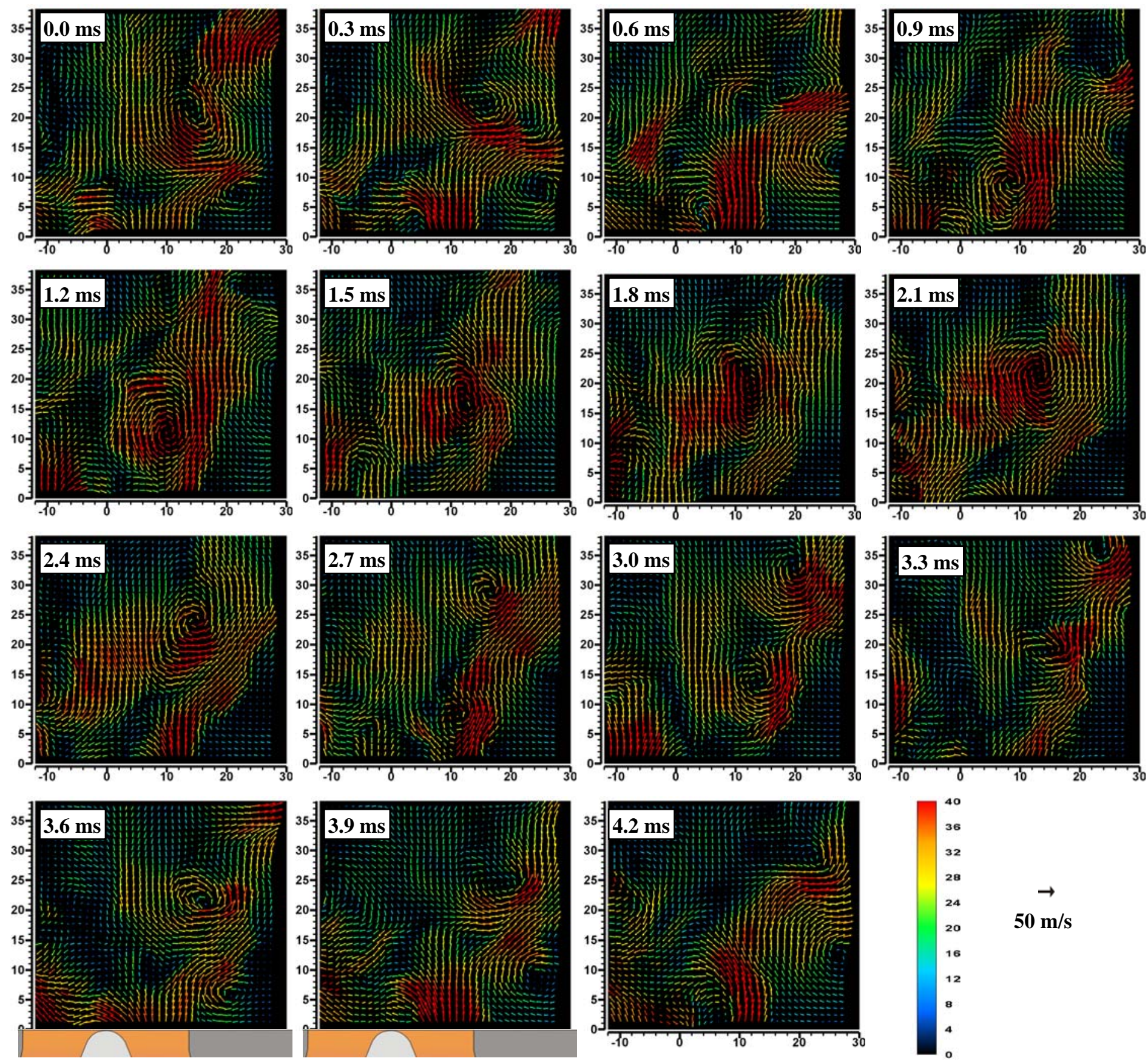

Fig.7 Image sequence from stereo PIV measurements in acoustically unstable flame of the Turbomeca burner. Every $3^{\text {rd }}$ frame of the $10 \mathrm{kHz}$ measurement is displayed. The vectors represent the planar velocity ( $\mathrm{x}$ and y component), the color bar represents the velocity magnitude (including also the $\mathrm{z}$ component). The flame axis is at $\mathrm{x}=0$. The nozzle exit is indicated in the frames at $\mathrm{t}=3.6$ and $3.9 \mathrm{~ms}$. 


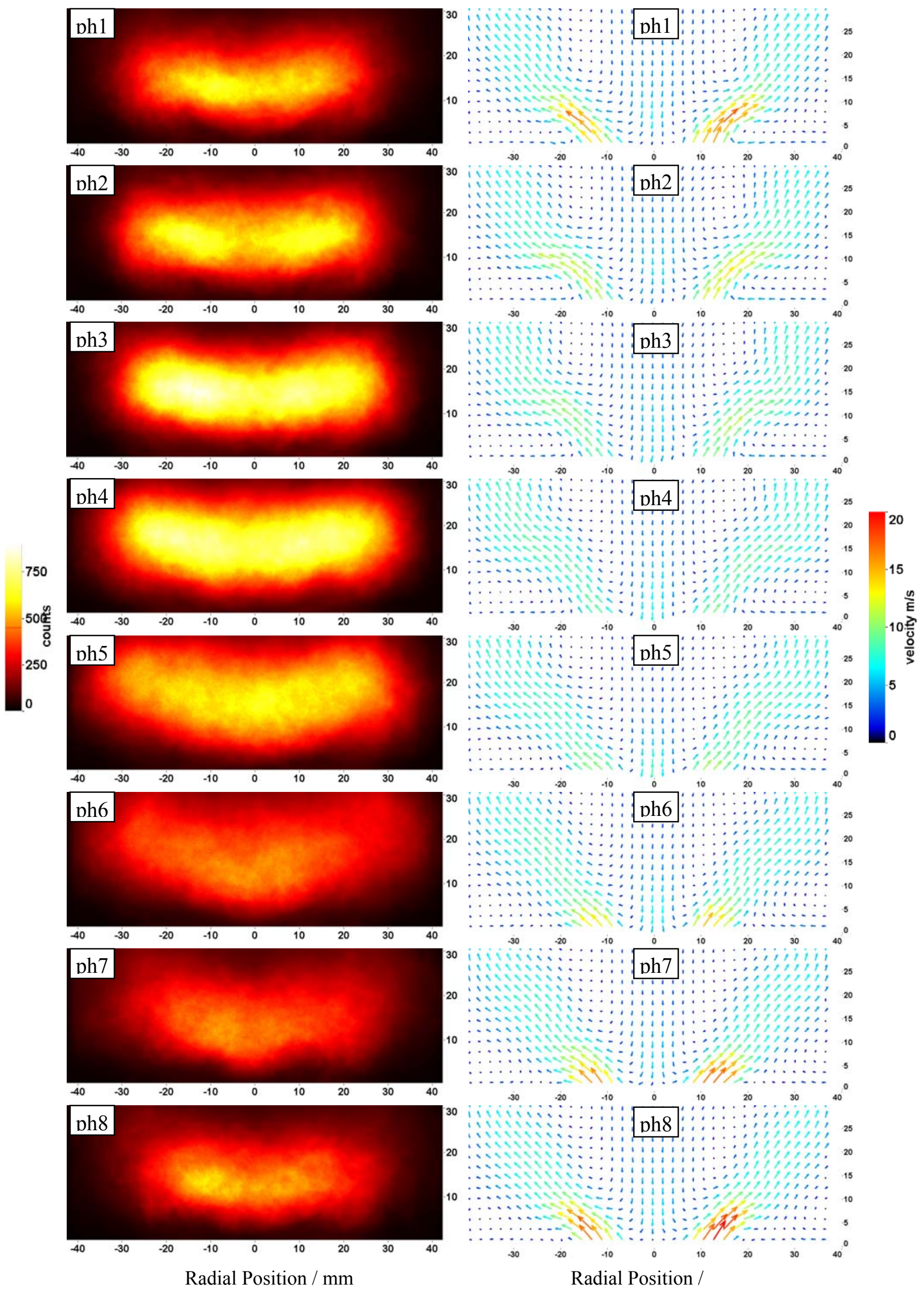

Fig.8 Phase-correlated mean distributions of the $\mathrm{OH}$ chemiluminescence and planar velocities from the dual swirl burner for 8 phase angles. 

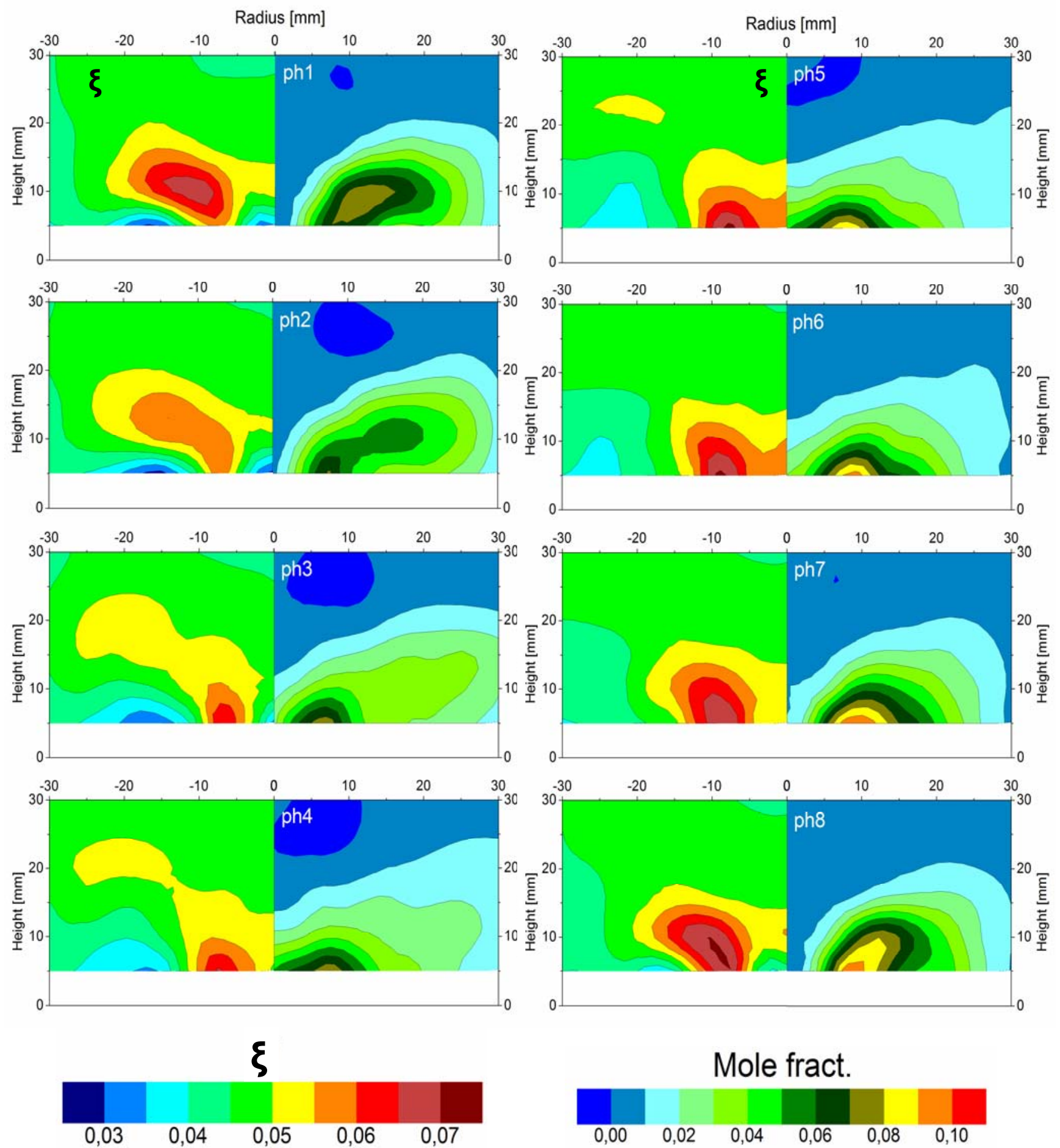

Mole fract.

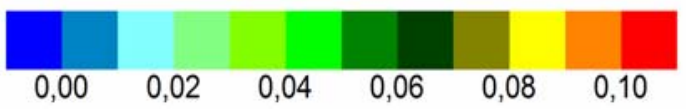

Fig.9 Phase-correlated mean distributions of mixture fraction (left half of each image) and $\mathrm{CH}_{4}$ mole fraction (right half of each image) from the dual-swirl burner. The results from Raman measurements in a grid of 45 locations have been interpolated to create 2D plots. 


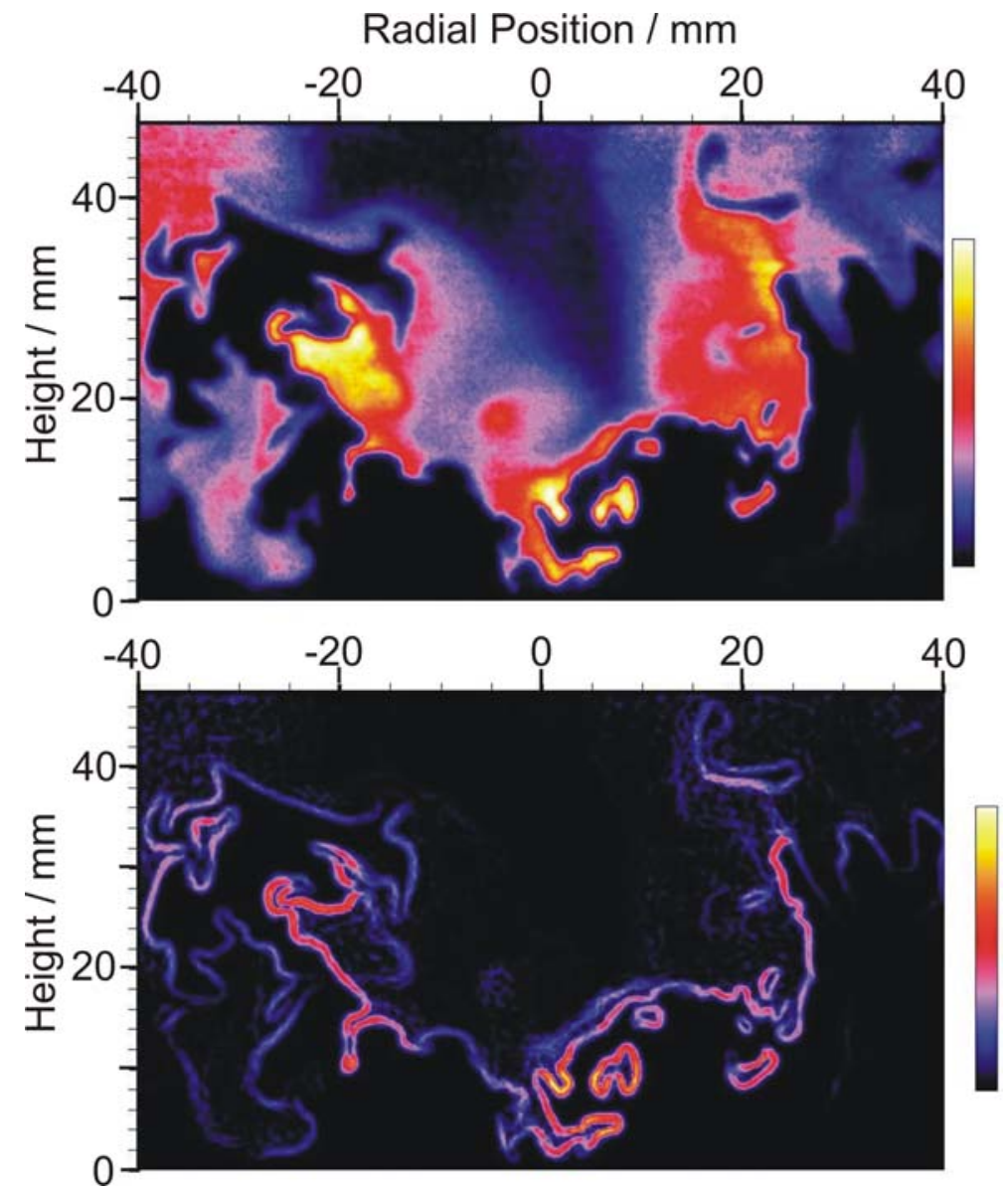

Fig.10 Single shot OH PLIF image (upper frame) recorded in the oscillating flame $\left(\mathrm{P}_{\mathrm{th}}=10\right.$ $\mathrm{kW}$ ) in the double swirl burner and corresponding gradients of the OH PLIF distribution. 

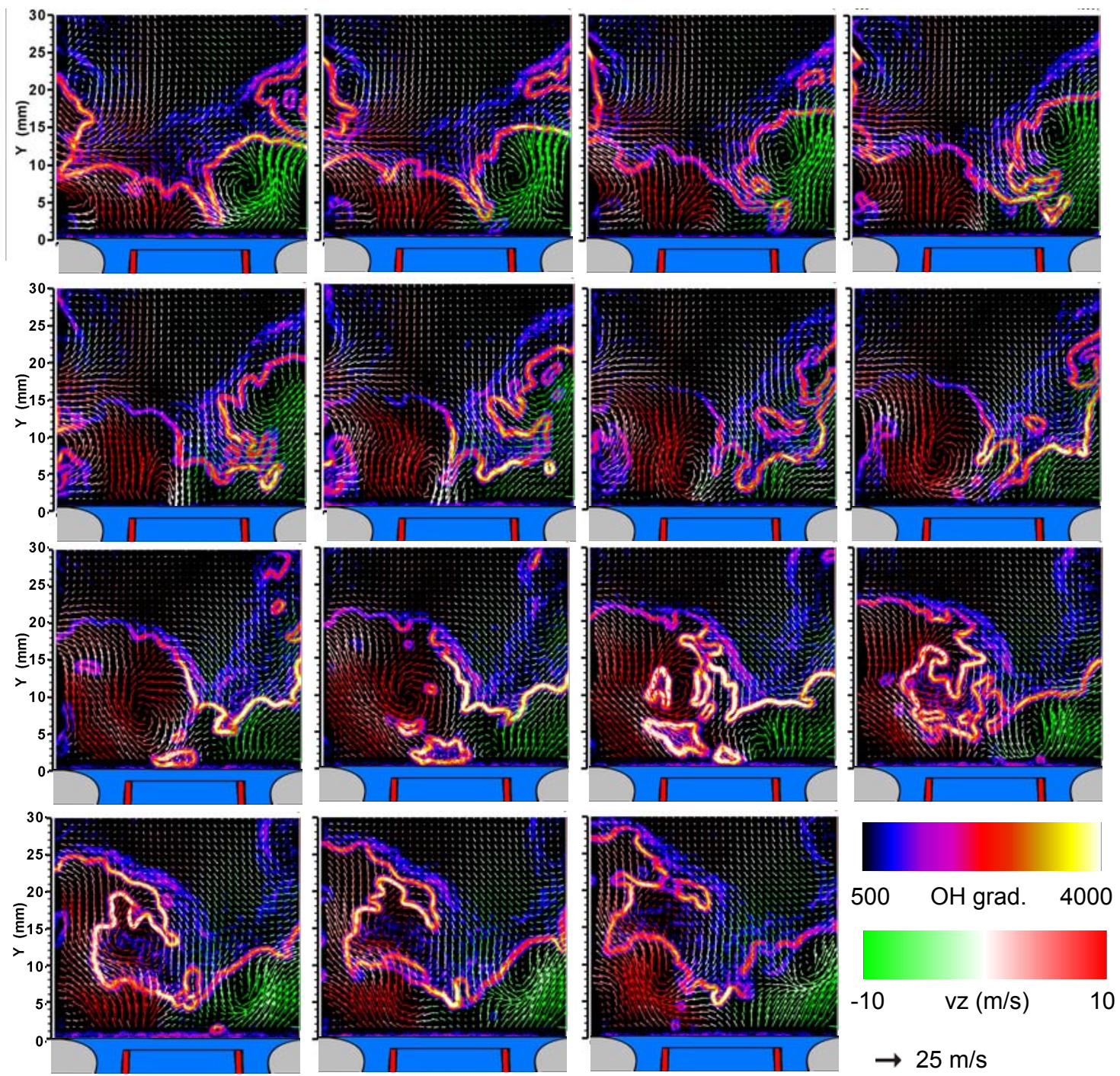

Fig.11 Image sequence from simultaneous OH PLIF/PIV measurements in the dual-swirl burnerat $5 \mathrm{kHz}$ frame rate. The flame front can be identified by the large gradients of the OH LIF distributions. Vectors show in-plane velocity components, the colors of the vectors indicate the out-of-plane velocity component according to the color bar. The field of view is $30 \mathrm{~mm} \times 30 \mathrm{~mm}$. The position and shape of the nozzle are shown below the distributions. 